Article

\title{
Biodiversity of Nematode Communities Associated with Wheat (Triticum aestivum L.) in Southern Morocco and Their Contribution as Soil Health Bioindicators
}

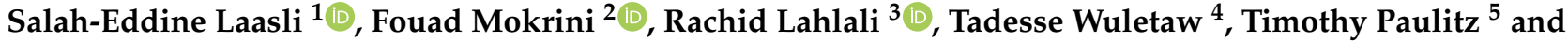 \\ Abdelfattah A. Dababat ${ }^{6, *(D)}$
}

1 Laboratory of Botany, Mycology, and Environment, Faculty of Science, Mohammed V University, Rabat 10080, Morocco; laaslisalaheddine@gmail.com

2 Biotechnology Unit, Regional Center of Agricultural Research, INRA-Morocco, Rabat 10080, Morocco; fmokrini.inra@gmail.com

3 Department of Plant and Environment Protection, Ecole Nationale d'Agriculture de Meknes, km. 10, Route Haj Kaddour, B.P. S/40, Meknès 50001, Morocco; rlahlali@enameknes.ac.ma

4 International Center for Agricultural Research in the Dry Areas (ICARDA), Experiment Station, INRA-Quich, Rue Hafiane Cherkaoui, Agdal, Rabat 10080, Morocco; w.tadesse@cgiar.org

5 Wheat Health, Genetics and Quality Research Unit, United States Department of Agriculture, Agricultural Research Service, Washington State University, Pullman, WA 99164-6430, USA; timothy.paulitz@usda.gov

6 International Maize and Wheat Improvement Center (CIMMYT), P.K. 39, Emek, Ankara 06511, Turkey

* Correspondence: a.dababat@cgiar.org

check for

updates

Citation: Laasli, S.-E.; Mokrini, F.; Lahlali, R.; Wuletaw, T.; Paulitz, T.; Dababat, A.A. Biodiversity of Nematode Communities Associated with Wheat (Triticum aestivum L.) in Southern Morocco and Their Contribution as Soil Health Bioindicators. Diversity 2022, 14, 194. https:/ / doi.org/10.3390/d14030194

Academic Editor: Michael Wink

Received: 16 February 2022

Accepted: 3 March 2022

Published: 7 March 2022

Publisher's Note: MDPI stays neutral with regard to jurisdictional claims in published maps and institutional affiliations.

Copyright: (C) 2022 by the authors. Licensee MDPI, Basel, Switzerland. This article is an open access article distributed under the terms and conditions of the Creative Commons Attribution (CC BY) license (https:// creativecommons.org/licenses/by/ $4.0 /)$.

\begin{abstract}
Soil nematodes are major soil organisms known to reduce wheat production worldwide. However, they are a crucial part of soil biodiversity. Due to the limited data available regarding nematode biodiversity associated with cereal crops in Morocco, a survey was conducted in 2019 across 80 wheat fields. Thirty-three nematode taxa were obtained with different trophic groups: 11 bacterivores (i.e., Rhabditis and Cephalobus spp.), 11 herbivores (i.e., Pratylenchus and Heterodera spp.), 6 fungivores (i.e., Ditylenchus and Aphelenchoides spp.), 4 omnivores (i.e., Dorylaimus and Aporcelaimus spp.) and one predator (Mononchus spp.). For each locality, the diversity of nematode communities was assessed through multiple diversity indices. Three areas (Ait Melloul, Oulad Dahou, and Sidi Boushab) were more diversified and had pronounced nematode occurrence. Moreover, few localities (e.g., Ait Amira and Lqliaa) were shown to be disturbed. Our study underlined that several nematode taxa were significantly correlated with edaphic and climatic/geographic properties. On the other hand, the spatial and parsimony analyses revealed that plant feeders were most associated with bacterial feeders in disturbed soils. This work revealed that the soil nematodes associated with wheat crops are diverse and can serve as an important tool for soil biomonitoring at a large scale.
\end{abstract}

Keywords: biodiversity; soil health; soil nematodes; trophic groups; wheat

\section{Introduction}

Wheat (Triticum spp.) is the most widely grown crop in the world, with more than 218 million ha, and its world trade is much greater than for all other crops combined [1]. Morocco is the world's 23rd wheat-producing country. Wheat is cultivated over 3.2 million ha and about 8 million tonnes were produced in the 2019-2020 growing season including 2.2 million ha of bread wheat and 1 million ha of durum wheat [1]. However, this production does not satisfy Moroccan needs and around 2-3 million tonnes (30\% more than average) is still imported from many countries in Europe and North America [1]. The low yield productivity is caused by several biotic and abiotic factors [2]. Among these factors, low availability of irrigation water, the low status of soil nutrients, as well as outbreaks of insect pests and diseases are major constraints that limit its production [3]. Plant-parasitic nematodes (PPNs) are one of the main biotic factors that attack and damage plant roots 
causing plant stress, ultimately leading to a significant yield loss [4-6]. In cereals, PPNs mostly belong to three groups, viz., cereal cyst nematodes (CCN, Heterodera spp.), rootlesion nematodes (RLN, Pratylenchus spp.), and root-knot nematodes (RKN, Meloidogyne spp.). These nematodes are distributed globally and implicated in significant economic yield loss [6].

Cereal cyst nematodes are placed among the oldest PPNs discovered genera [7]. It has been well documented throughout wheat-producing countries around the world as causing tremendous economic yield loss [4,5,7-10]. H. avenae is the most widely distributed and damaging species of $\mathrm{CCN}$ in many countries worldwide including North Africa, Europe, Australia, the USA, and West Asia [4,7-11]. H. latipons, the Mediterranean cereal cyst nematode has been reported in the Mediterranean region [12], Europe [13], and Central Asian Republics [14] in which temperate continental climates frequently occur. Recently, Mokrini et al. [11] reported the presence of H. avenae in Sais, Zaers, and Chaouia regions of Morocco. Whereas H. latipons has been reported for the first time from the Sais region of Morocco [15].

Root lesion nematodes (Pratylenchus spp.) are widespread and considered one of the most important groups of PPNs in the world due to their wide host range $[10,16,17]$ which makes their control extremely difficult using crop rotation [18]. In Morocco, Pratylenchus spp. constitute the most important group of PPNs in different wheat-growing areas $[19,20]$. They cause extensive necrosis on the roots with consequent crop losses. Four species, viz., P. penetrans, P. thornei, P. pseudocoffeae, and P. pinguicaudatus, have been detected in different wheat-growing areas [20].

The root-knot nematode (Meloidogyne artiellia) is an important PPN that cause damage to cereal crops, including wheat (Triticum durum and T. vulgare), barley (Hordeum vulgare), and sorghum (Sorghum vulgare), and occurs in the European, Middle Eastern, and Mediterranean Basin countries, including North Africa [6]. Its development and infectivity are strictly associated with climatic conditions (low temperatures). Although the root galls induced by M. artiellia are relatively small, the highly infested plants show chlorotic leaves and poor growth [6]. Other species, viz., M. incognita, M. naasi, M. graminicola, and M. chitwoodi could be prevalent in infested cereal fields [6].

Diversity and soil-related functions have recently led to the concept of "soil quality" defined by its ability to function within a given ecosystem to sustain organic production, maintain environmental quality, and promote plant health [21]. The knowledge of the structure of the nematode community provides information related to the different processes performed in the soil, the food web in the soil, and the state of stability of agro-ecosystems and soil biodiversity $[22,23]$. The set of nematode characteristics as bio-indicators of agricultural soils is relevant for the study of environmental changes [24]. Moreover, soil nematodes have been proven to play a major role in improving the physical properties of the soil, in which they can promote carbon and nitrogen transformation and maintain ecosystem health by contributing to the soil food web [24]. Several authors have shown that the nematode community can be used to evaluate soil quality $[25,26]$. Therefore, understanding the mechanisms behind nematode biodiversity, especially on a large scale is of great importance to establish adequate extensive programs for soil and plant health and for maintaining sustainable agriculture [27].

The soil nematode communities have been the subject of several studies to estimate the impact of some disturbances on the biological activity of soils using biodiversity indices. Neher et al. [28] indicated that the best soil health and quality indicators are based on the structure of nematode communities. Similarly, the use of plant-parasitic and predatory nematodes serve as indicators of human influence and activity on soil [29] and its physicochemical characteristics [30]. However, the study of soil nematode communities remains neglected and knowledge of the effects of cultural practices on these communities remains poorly documented. In Morocco, most research in this area has focused solely on PPNs communities, and to our knowledge, data on other nematode communities and their use as bio-indicators for assessing soil quality have not been reported yet. Therefore, the objectives 
of this study were to (i) investigate all soil nematode communities in South Moroccan wheat agro-systems, (ii) evaluate the potential of nematodes as potential bioindicators of soil quality and health in wheat fields, and (iii) predict the spatial contribution and succession of each nematode trophic levels in regards of soil health attributes.

\section{Materials and Methods}

\subsection{Nematode Sampling Design}

An intensive survey was established to investigate the diversity of nematode communities in different wheat agro-systems in the Southern regions of Morocco. The surveyed fields were selected based on their contribution to total production, soil type, and geographic distribution. Samples were taken during the wheat heading growth stage from 10 provinces accounting for 80 representative fields (Figure 1; Table 1). Fifteen subsamples were randomly collected in a zigzag pattern from the top $25 \mathrm{~cm}$ soil layer across an area of 1 to 2 ha using an auger ( $25 \mathrm{~mm}$ diameter) then mixed thoroughly to form a composite sample of $2 \mathrm{~kg}$ (soil and roots). Polyethylene bags were used to store samples at $4{ }^{\circ} \mathrm{C}$ to minimize nematode population changes and prevent water loss [31]. Nematode extraction and identification were carried out in the Nematology Laboratory at the National Institute of Agronomic Research (INRA-Agadir, Morocco).

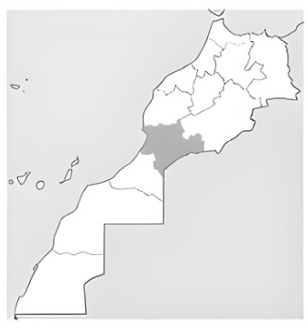

Legend

Ait Amira (10) Ait Melloul (8) Belfaa (8) Biongra (12) Lqliaa (7)

Massa (5)

Oulad Dahou (6) Sidi Bibi (11)

Sidi Boushab (9) Temsia (4)

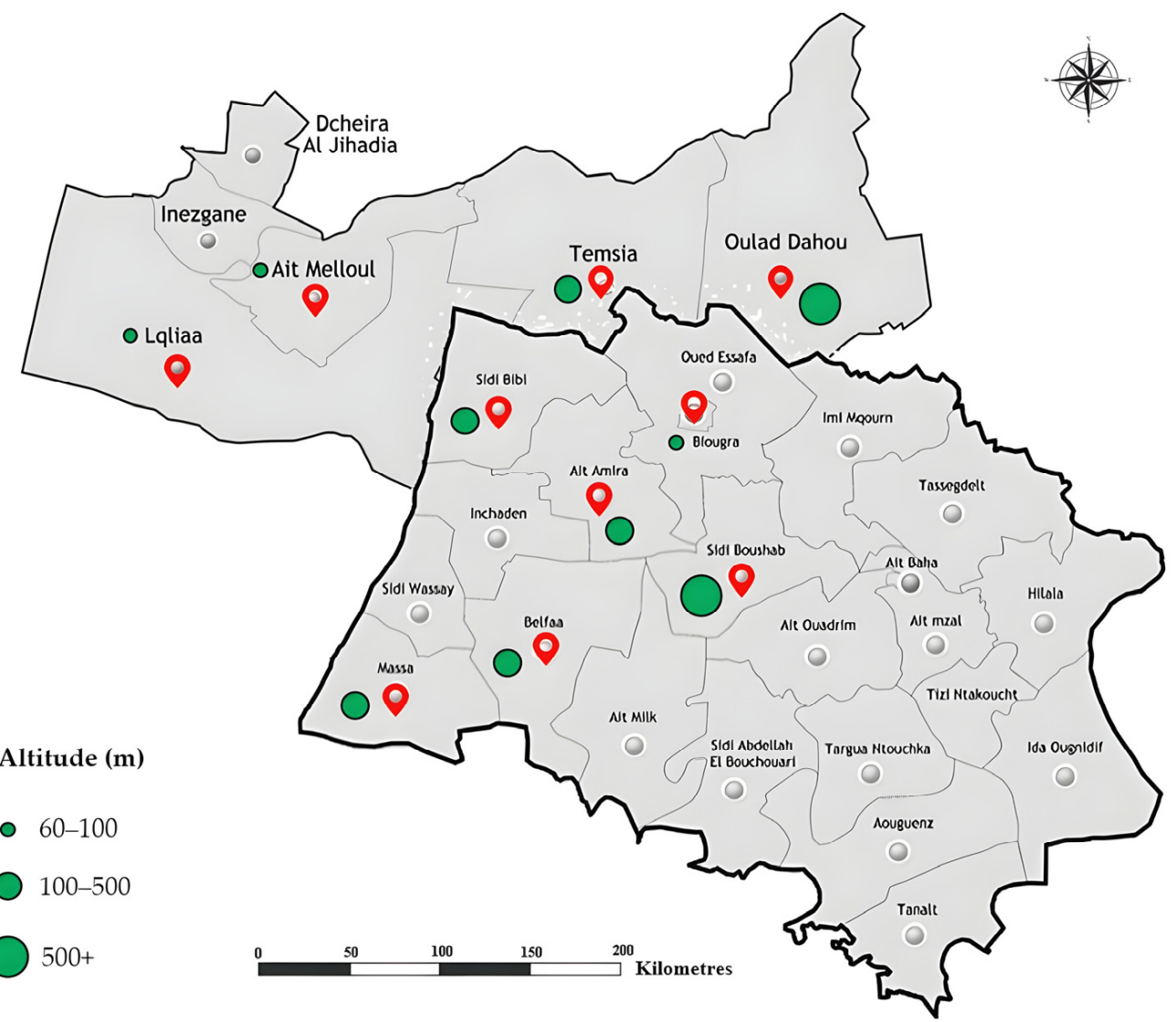

Figure 1. Map of distinct localities surveyed for nematodes in the Southern region of Morocco. 
Table 1. Locations and characteristics of sampling sites of the surveyed cereal-growing regions in Southern Morocco.

\begin{tabular}{cccccc}
\hline Locality (Province) & GPS (Latitude, Longitude) & Soil Texture & Organic Matter & pH & Samples per Site \\
\hline Ait Amira (AA) & $+30^{\circ} 10^{\prime} 35.4^{\prime \prime} \mathrm{N}, 9^{\circ} 29^{\prime} 18.96^{\prime \prime}$ & Sandy clay loam & $1.7 \%$ & 8.5 & 10 \\
Ait Melloul (AM) & $+30^{\circ} 20^{\prime} 3^{\prime \prime} \mathrm{N}, 9^{\circ} 29^{\prime} 50^{\prime \prime}$ & Medium loam & $2.7 \%$ & 9.3 & 8 \\
Belfaa (BE) & $+30^{\circ} 1^{\prime} 53^{\prime \prime} \mathrm{N}, 9^{\circ} 33^{\prime} 15^{\prime \prime}$ & Sandy loam & $2.0 \%$ & 8.1 & 8 \\
Biougra (Bi) & $+30^{\circ} 12^{\prime} 52^{\prime \prime} \mathrm{N}, 9^{\circ} 22^{\prime} 15^{\prime \prime}$ & Clay loam & $2.5 \%$ & 8.5 & 12 \\
Lqliaa (Lq) & $+30^{\circ} 17^{\prime} 27^{\prime \prime} \mathrm{N}, 9^{\circ} 27^{\prime} 58^{\prime \prime}$ & Sandy loam & $1.9 \%$ & 7.4 & 7 \\
Massa (Ma) & $+30^{\circ} 02^{\prime} 00^{\prime \prime} \mathrm{N}, 9^{\circ} 38^{\prime} 00^{\prime \prime}$ & Clay loam & $2.3 \%$ & 8.3 & 5 \\
Oulad Dahou (OD) & $+30^{\circ} 21^{\prime} 42.6^{\prime \prime}, 8^{\circ} 35^{\prime} 51.50^{\prime \prime}$ & Sandy clay loam & $1.9 \%$ & 8.6 & 6 \\
Sidi Bibi (SBi) & $+30^{\circ} 14^{\prime} 0^{\prime \prime} \mathrm{N}, 9^{\circ} 32^{\prime} 0^{\prime \prime}$ & Medium loam & $2.6 \%$ & 9.4 & 11 \\
Sidi Boushab (Sbo) & $+30^{\circ} 4^{\prime} 26.4^{\prime \prime} \mathrm{N}, 9^{\circ} 16^{\prime} 44.4^{\prime \prime}$ & Sandy loam & $1.7 \%$ & 7.6 & 9 \\
Temsia (TE) & $+30^{\circ} 21^{\prime} 36^{\prime \prime} \mathrm{N}, 9^{\circ} 24^{\prime} 50.4^{\prime \prime}$ & Clay loam & $2.3 \%$ & 7.7 & 4 \\
\hline
\end{tabular}

\subsection{Nematode Processing}

Nematode extraction was carried out via a modified Baermann technique [32] for both soil $\left(100 \mathrm{~cm}^{3}\right)$ and root $(20 \mathrm{~g})$ matrices. Nematode suspension was collected and processed under a stereomicroscope (Olympus $\mathrm{CH}-2$; Japan) after $72 \mathrm{~h}$ of incubation. Nematodes were killed and fixed by adding $4 \%$ hot formaldehyde $\left(60-80{ }^{\circ} \mathrm{C}\right)$ to a small drop of water in a glass cavity vessel. The nematodes were transferred to solution I (formaldehyde $(4 \%)$ + pure glycerin $(99: 1 ; v / v)$ ) and then placed in a desiccator containing about $1 / 10$ th of its volume of ethanol (96\%). After $24 \mathrm{~h}$, the watch glass containing the nematodes was removed from the desiccator and incubated at $37^{\circ} \mathrm{C}$. Then, $3 \mathrm{~mL}$ of solution II (ethanol $(96 \%)+$ pure glycerin $(95: 5 ; v / v))$ was added. This was repeated three times at $3 \mathrm{~h}$ intervals, while the watch glass was partially covered by a glass slide for evaporation. Finally, $2 \mathrm{~mL}$ of solution III (ethanol $(96 \%)+$ pure glycerin $(50: 50 ; v / v)$ ) was added and the watch glass was incubated and left overnight at $37^{\circ} \mathrm{C}$ [33]. Soil nematodes were identified to genus level using dichotomous keys [34,35] based on morphological features including body traits, stylet type, lip region, head shape, pharyngeal overlap, tail type, spermatheca shape, and vulva position. Subsequently, nematode specimens were prepared via the glycerin-ethanol technique for species-level identification and were then observed under a light microscope (Nikon Eclipse E200, Tokyo, Japan) using various identification keys [36-39]. Species of rootknot nematodes (RKNs) were identified via the establishment of the perineal patterns [40]. Adult females were carefully removed from root tissues and kept in a solution of sodium chloride $(0.9 \%)$ for $2 \mathrm{~min}$. Neck and lip regions were eliminated, and the posterior end was cleared. The perineal patterns were trimmed and transferred to a drop of glycerin for microscopic assessment ( $\times 100$ magnification). For cereal cyst nematodes (CCNs), vulval cones were established for identification from brown cysts alongside permanent microscope slides from second-stage juveniles (J2) fixed in TAF solution and embedded in glycerin [41]. Second-stage juveniles were recovered from cysts kept in water used for morphological identification.

\subsection{Soil Physico-Chemical Analyses}

Soil physico-chemical analyses were performed at the soil laboratory of INRA-Agadir, using regular methods [42]. Soil texture proprieties including clay (Cla; $0-2 \mu \mathrm{m}$ ), silt (Sil; 2-50 $\mu \mathrm{m}$ ), sand (San; 50 to $>200 \mu \mathrm{m}$ ), and Limestone (Lim) proportions were thoroughly analyzed. The electrical conductivity EC $(\mu \mathrm{S} / \mathrm{cm})$ and $\mathrm{pH}$ were determined via the 1:2.5 soil: water ratio technique [43]. As for the mineral content, the rates of potassium (K), manganese $(\mathrm{Mn})$, and magnesium $(\mathrm{Mg})$ alongside the exchangeable acidity were distinguished via atomic absorption spectrometry [44]. The soil organic matter rate (humic (Hom) and total (Tom)) was processed using the method described by Allison [45] while carbon (C) and nitrogen $(\mathrm{N})$ contents were measured using Walkley-Black [46] and Kjeldahl methods [47], respectively. The carbon to nitrogen ratio $(\mathrm{C}: \mathrm{N})$ was also calculated. Other components including iron $(\mathrm{Fe})$, copper $(\mathrm{Cu})$, zinc $(\mathrm{Zn})$, calcium $(\mathrm{Cal})$ sodium $(\mathrm{Na})$, and phosphorus 
(P) were processed using colorimetry methods (Shimadzu UV-1205; Shimadzu Scientific Instruments, Columbia, MD, USA).

\subsection{Diversity Assessment of Nematodes Taxa}

Nematode incidence was determined by determining the prevalence and maximum density [48] for each nematode feeding type. Prevalence was obtained as the percentage of samples with well-defined nematode taxon using the following equation:

$$
\text { Prevalence }(\%)=\text { Sn } / \text { St } \times 100
$$

where Sn considers samples having the desired nematode taxon, and St is the total of samples processed.

For more advanced biodiversity screening, several ecological and biomonitoring indices were measured to assess nematode diversity and structure in each locality surveyed. These indices included Shannon-Weaver diversity index $\mathrm{H}^{\prime}=-\sum_{\mathrm{i}-1}^{\mathrm{s}} \mathrm{pi} \ln \mathrm{pi}$, where $s$ is the number of nematode taxa, pi is the taxa proportion [49]; the evenness $\mathrm{J}=\mathrm{H} / \mathrm{Hmax}, \mathrm{Hmax}=\log \_\mathrm{s}$; nematodes maturity index $(\mathrm{MI})=\sum(\mathrm{vi}) \times(\mathrm{fi})$, where vi comprises the $c-p$ values of nematode taxon $i$ according to their $r$ and $k$ properties, and fi is the frequency of taxon $i$ in a sample; plant-parasitic index (PPI) which was determined similarly except for replacing $c-p$ values to $p-p$ values [22]; channel index $(\mathrm{CI})=100 \times(0.8 \mathrm{Fu} 2 /(3.2 \mathrm{Ba} 1+0.8 \mathrm{Fu} 2)$ which indicates decomposition nature; Enrichment index $(E I)=100 \times\left(\sum k_{e} n_{e} /\left(k_{e} n_{e}+\sum k_{b} n_{b}\right)\right)$, where $k_{b}$ is the assigned weight to the functional guilds $\mathrm{Ba} 2$ and $\mathrm{Fu} 2$, and $\mathrm{n}_{\mathrm{b}}$ represents their corresponding abundance, which determines basal food web characteristics, $k_{e}$ is the assigned weight to $\mathrm{Ba} 1$ and $\mathrm{Fu} 2$ guilds, and $\mathrm{n}_{\mathrm{e}}$ corresponds to their abundance; and structure index $(\mathrm{SI})=100 \times\left(\sum \mathrm{k}_{\mathrm{s}} \mathrm{n}_{\mathrm{s}} /\left(\mathrm{k}_{\mathrm{s}} \mathrm{n}_{\mathrm{s}}+\sum \mathrm{k}_{\mathrm{b}} \mathrm{n}_{\mathrm{b}}\right)\right)$ where $\mathrm{k}_{\mathrm{s}}$ is the assigned weight to guilds Ba3-Ba5, Fu3-Fu5, Om4-Om5, and Pr2-Pr5, and $\mathrm{n}_{\mathrm{s}}$ corresponds to their abundance, representing food web structural attributes [50].

\subsection{Statistical Analyses}

To explore nematodes' community and soil physical-chemical patterns from different wheat agro-systems surveyed, principal component analyses (PCA) were conducted. Nematode and soil variables linked with the PCA were subjected to a two-way ANOVA after the normalization session of the data using the Anderson-Darling normality test [51], performed using XLSTAT 2016.02.28451 software (Addinsoft, USA). Significant differences among variables were performed using Fisher's protected least significant difference (LSD) and Tukey test at $p<0.05$. Differences obtained at levels of $p<0.05$ were viewed to be significant. Taxon dominance parameter was identified for each nematode taxa in the surveyed localities alongside their frequencies. The distribution diagram of nematodes communities was applied as abundance variables were transformed to $\log _{10}(X+1)$ before analysis [52]. Food web and metabolic footprints analysis was conducted using NINJA (Nematode INdicator Joint Analysis) software (https:/ / sieriebriennikov.shinyapps.io/ninja/) (accessed on 4 January 2021) [53]. Advanced heatmap analysis (heatmap-2) was adopted to visualize correlations between nematodes and soil physical-chemical properties, based on Ward's algorithm of R software [54]. To meet normality assumptions, nematode variables were square-root transformed before heatmap analysis. All multivariate analyses were conducted using R software. Nematode spatial contribution and parsimony mapping network (PMN) analyses were conducted using BioPython matplotlib library with Yellowbrick machine learning module [55] and sviewgui PyQt-based GUI packages [56] alongside Bio.Phylo module in PyCharm API [57] and NetworkX library [58], respectively. The unidimensional plot that projected the contribution of nematodes was generated according to the ANOSIM (Analysis of similarities) and SIMPER (Similarity percentages) analyses [51]. Venn diagrams and lollipop plots were conducted using BioPython Matplotlib-venn [59] and Matplotlib-lollipop [60] modules. Redundancy analysis (RDA) was conducted using Python matplotlib and ordination libraries (skbio.stats.ordination) [61]. 


\section{Results}

\subsection{Nematode Community Patterns and Ecological Distribution in Wheat Fields}

The main idea of this study was to investigate the diversity of soil nematodes alongside their potential as soil bio-indicators through conducting an extensive survey across different cereal fields in the central southern Moroccan region. The obtained results showed that 33 nematode genera were identified from this survey (Table 2). Regarding their trophic level, the fraction of soil nematodes was calculated (Figure 2). Plant-parasitic nematodes (herbivores) were accurately represented in all localities with $40 \%$ of occurrence, followed by bacterivores (30\%), fungivores (20\%), both omnivores and predators (10\%) (Figure $2 \mathrm{a}$ ). The projection of free-living nematodes showed that bacterivorous nematodes were predominant in the majority of wheat-producing areas $(>60 \%)$ compared to the other feeding types (Figure $2 b$ ). The fraction of herbivorous nematodes varied according to parasitism type. Migratory endoparasites (e.g., root-lesion nematodes, Pratylenchus spp.), ectoparasites (e.g., Longidorus spp.), sedentary parasites (e.g., Heterodera spp.), and root hair feeders (e.g., Tylenchus spp.) occurred throughout all localities sampled (Figure 2c). Furthermore, these nematodes were classified according to c-p (colonizer-persister) values for the free-living organisms, and to $\mathrm{p}-\mathrm{p}$ (plant-parasitic) values for herbivorous nematodes. The predominance of c-p 2 nematodes was observed in all fields. However, superior c-p nematodes (c-p 4 and c-p 5) were also frequent (Figure 2d). For the p-p values, most nematodes were shown to be in second and third levels (p-p 2 and p-p 3) (Figure 2e).

Table 2. Main nematode taxa identified from wheat-growing areas in southern Morocco.

\begin{tabular}{|c|c|c|c|c|c|c|}
\hline Family & Genus & Trophic Group & c-p Class & p-p Class & Functional Guild & Code \\
\hline Rhabditidae & Rhabditis & Bacterivores & 1 & 0 & Ba1 & Rha \\
\hline \multirow[t]{4}{*}{ Cephalobidae } & Acrobeles & Bacterivores & 2 & 0 & $\mathrm{Ba} 2$ & Acr \\
\hline & Cervidellus & Bacterivores & 2 & 0 & $\mathrm{Ba} 2$ & Cer \\
\hline & Eucephalobus & Bacterivores & 2 & 0 & $\mathrm{Ba} 2$ & Euc \\
\hline & Cephalobus & Bacterivores & 3 & 0 & $\mathrm{Ba} 3$ & Cep \\
\hline Metateratocephalidae & Metateratocephalus & Bacterivores & 2 & 0 & $\mathrm{Ba} 2$ & Met \\
\hline Diplogasteridae & Diplogasteriana & Bacterivores & 1 & 0 & Ba1 & Dip \\
\hline Panagrolaimidae & Panagrolaimus & Bacterivores & 1 & 0 & Ba1 & Pan \\
\hline Plectidae & Plectus & Bacterivores & 2 & 0 & $\mathrm{Ba} 2$ & Ple \\
\hline \multirow[t]{2}{*}{ Prismatolaimidae } & Prismatolaimus & Bacterivores & 3 & 0 & $\mathrm{Ba} 3$ & Pri \\
\hline & Tylocephalus & Bacterivores & 2 & 0 & $\mathrm{Ba} 2$ & Tylo \\
\hline Aphelenchoididae & Aphelenchoides & Fungivores & 2 & 0 & $\mathrm{Fu} 2$ & Aph \\
\hline Aphelenchidae & Aphelenchus & Fungivores & 2 & 0 & $\mathrm{Fu} 2$ & Aphe \\
\hline Mydonomidae & Dorylaimoides & Fungivores & 4 & 0 & $\mathrm{Fu} 4$ & Dory \\
\hline Anguinidae & Ditylenchus & Fungivores & 2 & 0 & $\mathrm{Fu} 2$ & Dit \\
\hline \multirow[t]{2}{*}{ Tylencholaimidae } & Tylencholaimus & Fungivores & 4 & 0 & $\mathrm{Fu} 4$ & Tylc \\
\hline & Tylencholaimellus & Fungivores & 4 & 0 & $\mathrm{Fu} 4$ & Tylen \\
\hline Dorylaimidae & Dorylaimus & Omnivores & 4 & 0 & Om4 & Dor \\
\hline Qudsianematidae & Eudorylaimus & Omnivores & 4 & 0 & Om4 & Eud \\
\hline Aporcelaimidae & Aporcelaimus & Omnivores & 5 & 0 & Om5 & Apo \\
\hline Thornematidae & Thornenema & Omnivores & 5 & 0 & Om5 & Tho \\
\hline Mononchidae & Mononchus & Predators & 4 & 0 & Pr4 & Mon \\
\hline
\end{tabular}


Table 2. Cont.

\begin{tabular}{|c|c|c|c|c|c|c|}
\hline Family & Genus & Trophic Group & c-p Class & p-p Class & Functional Guild & Code \\
\hline Meloidogynidae & Meloidogyne & Herbivores & 0 & 3 & $\mathrm{He} 3$ & $\mathrm{Mel}$ \\
\hline Pratylenchidae & Pratylenchus & Herbivores & 0 & 3 & $\mathrm{He} 3$ & Pra \\
\hline Paratylenchidae & Paratylenchus & Herbivores & 0 & 2 & $\mathrm{He} 2$ & Par \\
\hline \multirow[t]{2}{*}{ Tylenchidae } & Tylenchus & Herbivores & 0 & 2 & $\mathrm{He} 2$ & Tyl \\
\hline & Psilenchus & Herbivores & 0 & 2 & $\mathrm{He} 2$ & Psi \\
\hline \multirow[t]{2}{*}{ Dolichodoridae } & Tylenchorhynchus & Herbivores & 0 & 3 & He3 & Tyle \\
\hline & Merlinius & Herbivores & 0 & 3 & $\mathrm{He} 3$ & Mer \\
\hline Longidoridae & Longidorus & Herbivores & 0 & 5 & $\mathrm{He} 5$ & Lon \\
\hline Heteroderidae & Heterodera & Herbivores & 0 & 3 & $\mathrm{He} 3$ & Het \\
\hline \multirow[t]{2}{*}{ Hoplolaimidae } & Hoplolaimus & Herbivores & 0 & 3 & $\mathrm{He} 3$ & Hop \\
\hline & Helicotylenchus & Herbivores & 0 & 3 & He3 & $\mathrm{Hel}$ \\
\hline
\end{tabular}

(a)

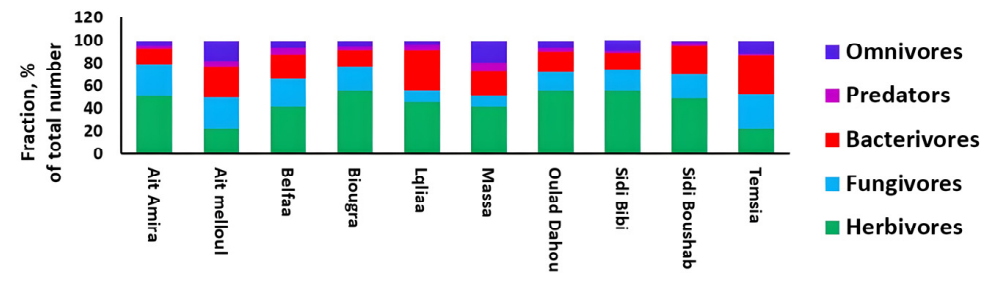

(b)

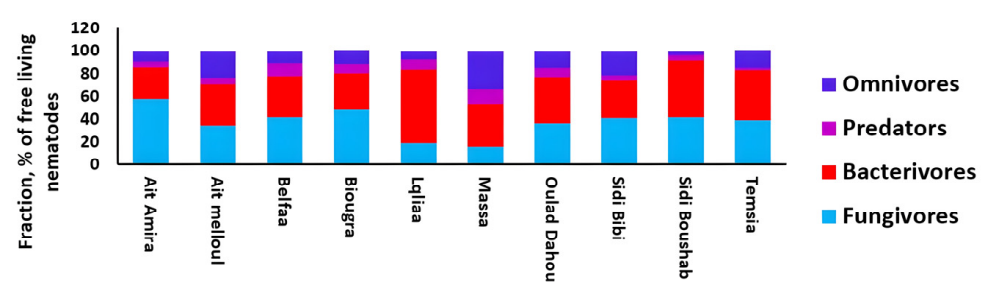

(c)

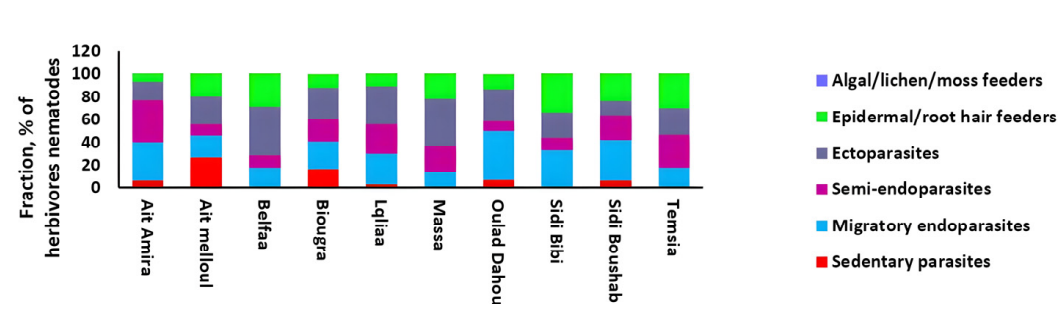

(d)

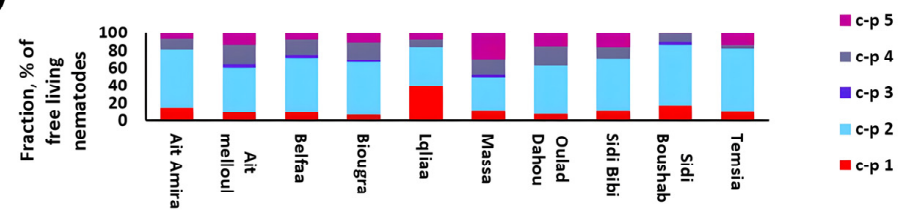

(e)

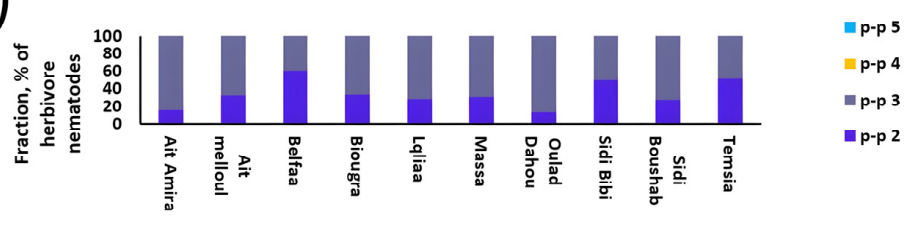

Figure 2. Fraction of soil nematode communities that occurred in wheat fields of Southern Morocco. (a) Fraction percentage of the total nematode numbers according to their respective feeding group. (b) Fraction of free-living nematodes. (c) Fraction of herbivorous (plant-parasitic) nematodes. (d) Fraction of free-living nematodes according to c-p (colonizer-persister) values. (e) Fraction of PPNs according to $\mathrm{p}-\mathrm{p}$ (plant-parasitic) values. 
The distribution patterns of the observed soil nematodes were analyzed for each locality (Figure 3). Principal component analyses of nematode genera distribution across the southern Moroccan region showed that the fraction of variance accounted for by the first two PC axes was $20.80 \%$ and $11.74 \%$ (eigenvalues) (Figure $3 \mathrm{a}$ ). The PC1 axis was related to 16 taxa including Eucephalobus, Cephalobus, Diplogasteriana, Aphelenchus, Dorylaimoides, Mononchus, and Merlinius spp. (positive PC values). The PC2 axis was related to 12 taxa including Cervidellus, Rhabditis, Prismatolaimus, Longidorus, Paratylenchus, and Hoplolaimus spp. (positive PC values). On the other hand, few nematode genera were associated with the negative axes of the PCA, which include Eudorylaimus, Panagrolaimus, and Tylenchus spp. This distribution pattern varied significantly across sampled localities. For instance, the highest nematode genera were occurred in Ouled Dahou, Massa, Lqliaa, and Sidi Boushab, while the lowest rates were observed in Ait Amira, Biougra, and Sidi Bibi (Figure 3b). Nematode abundance and frequency in each surveyed locality were computed (Figure 3c, Table 3). Out of the 33 taxa identified, 15 were shown to be dominant, including Pratylenchus, Helicotylenchus, Aphelenchoides, Heterodera, Plectus, Acrobeles, Cephalobus, and Tylenchorynchus spp. In addition, 6 taxa were frequent throughout prospected areas, including Merlinius, Meloidogyne, Mononchus, and Dorylaimoides spp. Occasional taxa were also obtained (e.g., Diplogasteriana, Longidorus, and Eucephalobus spp.). On the other hand, 7 taxa were depicted to have rare occurrences such as Metateratocephalus, Thornenema, Tylencholaimellus, and Tylocephalus spp. (Table 3).

(a)

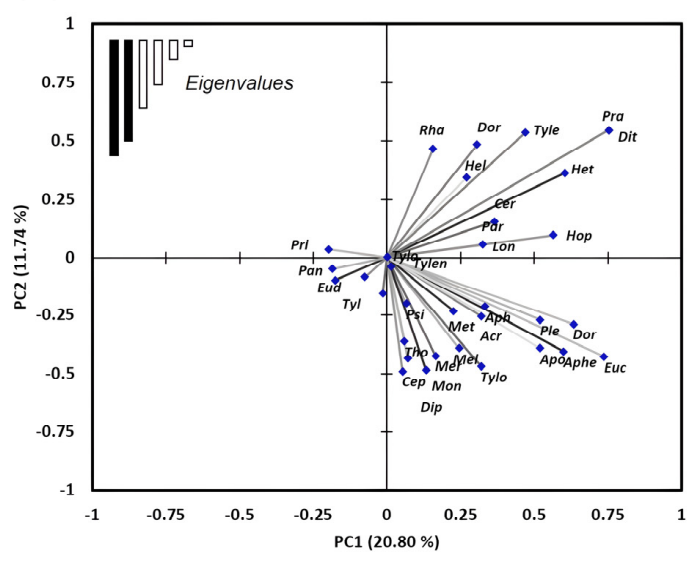

(b)

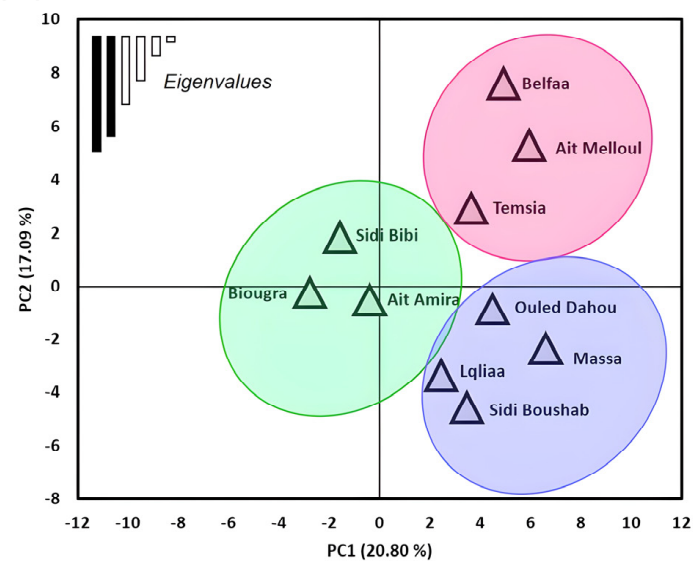

(c)

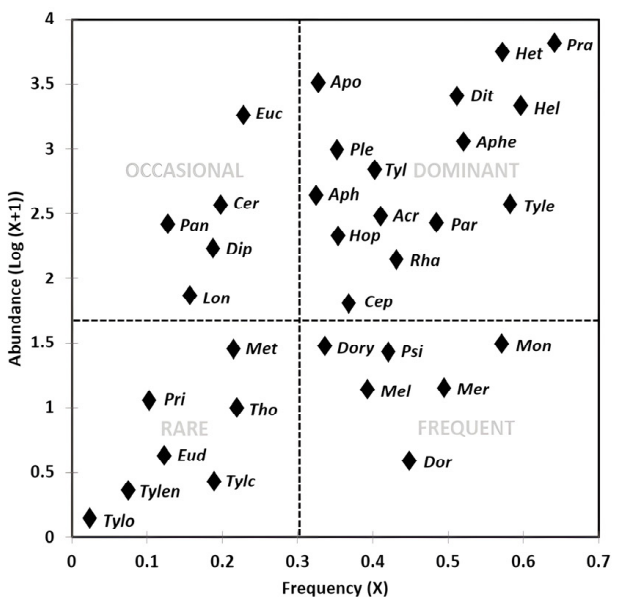

Figure 3. Nematode community patterns and distribution in sampled wheat fields of Southern Morocco. (a) Principal component analysis (PCA) (Kendall type) depicting nematode distribution patterns. (b) PCA of nematode distribution according to the different surveyed localities. (c) Distribution diagram (abundance-frequency) of nematode communities. 
Table 3. Mean abundance values $\left(100 \mathrm{~cm}^{3} \text { soil }\right)^{-1}$ for all nematodes identified from wheat-growing areas in southern Morocco.

\begin{tabular}{|c|c|c|c|c|c|c|c|c|c|c|c|}
\hline \multirow{2}{*}{ TG } & \multirow{2}{*}{ Nematode Taxa } & \multicolumn{10}{|c|}{ Localities * } \\
\hline & & AA & AM & BE & BI & LQ & MA & OD & SBi & SBo & TE \\
\hline \multirow{11}{*}{ 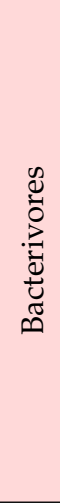 } & Rhabditis & $25.9 c$ & $45.6_{\mathrm{ab}}$ & $15.3 \mathrm{~d}$ & $7.5 \mathrm{e}$ & $57.8 \mathrm{a}$ & $15_{\mathrm{d}}$ & $35.4_{b}$ & $22_{c}$ & $15_{\mathrm{d}}$ & $55.5 \mathrm{a}$ \\
\hline & Acrobeles & $10 \mathrm{~d}$ & 48.96 a & $35_{b}$ & $15_{d}$ & $15.2 \mathrm{~d}$ & $0_{\mathrm{f}}$ & $22.3_{c}$ & $20_{c}$ & $7.5 \mathrm{e}$ & $50.3 \mathrm{a}$ \\
\hline & Cervidellus & $0_{\mathrm{d}}$ & $26.1 \mathrm{a}$ & $0_{\mathrm{d}}$ & $3.75 \mathrm{~cd}$ & $5.7 \mathrm{c}$ & $0_{\mathrm{d}}$ & $0_{\mathrm{d}}$ & $2.25 \mathrm{~cd}$ & $7.75 \mathrm{c}$ & $15_{b}$ \\
\hline & Eucephalobus & $0_{\mathrm{d}}$ & $35 \mathrm{a}$ & $5.6_{c}$ & $0_{\mathrm{d}}$ & $0_{\mathrm{d}}$ & $0_{\mathrm{d}}$ & $0_{\mathrm{d}}$ & $5.1_{c}$ & $2.25 \mathrm{~cd}$ & $13.9_{b}$ \\
\hline & Cephalobus & $20.6 \mathrm{bc}$ & 15.9 c & $14.4_{\mathrm{c}}$ & $9 \mathrm{~d}$ & $17_{c}$ & $33_{b}$ & $30.2 \mathrm{~b}$ & $10.6_{c}$ & $22.5 \mathrm{bc}$ & $80 \mathrm{a}$ \\
\hline & Metateratocephalus & $0_{c}$ & $20.3 a$ & $5.2 \mathrm{~b}$ & $0_{c}$ & $0_{c}$ & $8_{b}$ & $0_{\mathrm{c}}$ & $0_{\mathrm{c}}$ & $3.75_{b}$ & $0_{c}$ \\
\hline & Diplogasteriana & $5.75_{b}$ & $5.5_{b}$ & $5_{b}$ & $4_{b}$ & $7.5 \mathrm{a}$ & $0_{c}$ & $4_{b}$ & $0_{\mathrm{c}}$ & $7.3 \mathrm{a}$ & $10.4 \mathrm{a}$ \\
\hline & Panagrolaimus & $5.3_{b}$ & $12.8 \mathrm{a}$ & $5.2_{b}$ & $5_{b}$ & $5_{b}$ & $7_{b}$ & $4_{b}$ & $5.3_{b}$ & $4_{b}$ & $0_{c}$ \\
\hline & Plectus & $8.65 c$ & $66.2 \mathrm{a}$ & $10.4_{c}$ & $28.6 \mathrm{bc}$ & $11.3_{c}$ & $25.6 \mathrm{bc}$ & $13.4_{\mathrm{c}}$ & $39.3 \mathrm{~b}$ & $0_{\mathrm{d}}$ & $33.4_{b}$ \\
\hline & Prismatolaimus & $0_{c}$ & $10 \mathrm{a}$ & $5_{b}$ & $3.75_{b}$ & $0_{c}$ & $1_{\mathrm{bc}}$ & $0_{c}$ & $0_{\mathrm{c}}$ & $0_{\mathrm{c}}$ & $0_{\mathrm{c}}$ \\
\hline & Tylocephalus & $0_{b}$ & $0_{b}$ & $0_{b}$ & $0_{b}$ & $0_{b}$ & $0_{b}$ & $0_{b}$ & $5.6 \mathrm{a}$ & $0_{b}$ & $0_{b}$ \\
\hline \multirow{6}{*}{ 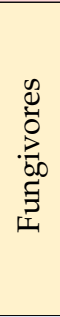 } & Aphelenchoides & $20.4_{c}$ & $130.3 \mathrm{a}$ & $25.1_{c}$ & $11.3 \mathrm{~d}$ & $15.1 \mathrm{~d}$ & $10 \mathrm{~d}$ & $10.2 \mathrm{~d}$ & $25_{c}$ & $15 \mathrm{~d}$ & $70_{b}$ \\
\hline & Aphelenchus & $11.6_{c}$ & $50.66 \mathrm{a}$ & $30.2_{b}$ & $26.5_{b}$ & $7.6_{\mathrm{d}}$ & $0_{\mathrm{e}}$ & $20 \mathrm{bc}$ & $20.3_{b c}$ & $3.75 \mathrm{~d}$ & $25_{b}$ \\
\hline & Dorylaimoides & $0_{\mathrm{d}}$ & $32.1 \mathrm{a}$ & $5.6_{c}$ & $7.5_{c}$ & $0_{\mathrm{d}}$ & $0_{\mathrm{d}}$ & $11.4_{\mathrm{b}}$ & $0_{\mathrm{d}}$ & $3.2_{c}$ & $5.5 \mathrm{c}$ \\
\hline & $\begin{array}{l}\text { Ditylenchus } \\
\text { D. dipsaci }\end{array}$ & $\begin{array}{l}98.3 \mathrm{a} \\
+\end{array}$ & $\begin{array}{l}22.8 \mathrm{c} \\
+\end{array}$ & $\begin{array}{l}55.7 \mathrm{~b} \\
+\end{array}$ & $\begin{array}{l}63.7 \mathrm{~b} \\
+\end{array}$ & $\begin{array}{l}15.9 \mathrm{~cd} \\
+\end{array}$ & ${ }_{-}^{0} \mathrm{~d}$ & $\begin{array}{l}25.8 \mathrm{c} \\
+\end{array}$ & $\begin{array}{l}35.1 \mathrm{bc} \\
+\end{array}$ & $\begin{array}{l}45.7 \mathrm{bc} \\
+\end{array}$ & $\begin{array}{l}65.6 \mathrm{~b} \\
+\end{array}$ \\
\hline & Tylencholaimus & $\begin{array}{l}+ \\
0_{c}\end{array}$ & $\begin{array}{l}+ \\
10.6\end{array}$ & $\begin{array}{l}+ \\
0_{c}\end{array}$ & ${ }^{+}$ & ${ }^{+}$ & $\overline{0}_{\mathrm{S}}$ & $a_{c}^{+}$ & 525 & + & $a^{+}$ \\
\hline & Tylencholaimellus & $0_{\mathrm{c}}$ & 5.3 & $0_{\mathrm{c}}$ & $0_{\mathrm{c}}$ & $0_{\mathrm{c}}$ & $0_{\mathrm{c}}$ & $0_{\mathrm{c}}$ & $0.25 \mathrm{~b}$ & $0_{c}$ & $u_{c}$ \\
\hline \multirow{4}{*}{ 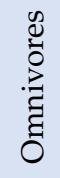 } & Dorylaimus & $12.4_{\mathrm{b}}$ & $22.9 a$ & 10.9 & $0^{d}$ & $0^{\mathrm{d}}$ & 4.4 & $\frac{0^{4}}{0}$ & 10 & 75 & 15 \\
\hline & Eudorylaimus & $0_{b}$ & $0_{\mathrm{b}}$ & & $3.75 \mathrm{a}$ & $0_{b}$ & $0_{\mathrm{b}}$ & $0_{b}$ & 5 & $0_{\mathrm{h}}$ & 5 \\
\hline & Aporcelaimus & $23.6 \mathrm{~cd}$ & $80.2 \mathrm{a}$ & $15.7 \mathrm{~d}$ & $30.5_{c}$ & $15.3 \mathrm{~d}$ & $26.7_{c}$ & $35.06_{c}$ & $25.6_{c}$ & $3.75 \mathrm{e}$ & $65.2_{b}$ \\
\hline & Thornenema & $0_{c}$ & $20.5 \mathrm{a}$ & $5.3_{b}$ & $0_{c}$ & $3.8_{\mathrm{b}}$ & $0_{c}$ & $0_{c}$ & $5.3_{b}$ & $0_{c}$ & $0_{c}$ \\
\hline \multirow[t]{12}{*}{ 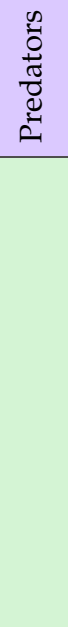 } & Mononchus & $15_{c}$ & $45.4 \mathrm{a}$ & $18.7 \mathrm{c}$ & $14.6_{c}$ & $30.2 \mathrm{~b}$ & $11_{c}$ & $20.8_{b}$ & $9.7 \mathrm{c}$ & $13_{c}$ & $5 \mathrm{~d}$ \\
\hline & Meloidogyne & $15.6 \mathrm{a}$ & $20.25 \mathrm{a}$ & $5.8_{c}$ & $7.5 \mathrm{c}$ & $3.75_{c}$ & $0_{d}$ & $11.2 \mathrm{~b}$ & $0_{d}$ & $11.3_{\mathrm{b}}$ & $0_{d}$ \\
\hline & M. incognita & + & + & - & + & + & - & + & - & - & - \\
\hline & M. javanica & + & - & + & - & - & - & - & - & + & - \\
\hline & M. arenaria & - & - & - & + & - & - & - & - & + & - \\
\hline & Pratylenchus & $70.8_{b}$ & $40.3_{c}$ & $35.4_{c}$ & $71.3_{b}$ & $48.6_{c}$ & $14.3 \mathrm{~d}$ & $70.4_{b}$ & $95.3 \mathrm{a}$ & $67.5_{b}$ & $45.05 \mathrm{c}$ \\
\hline & P. thornei & + & + & + & + & + & + & + & + & + & + \\
\hline & P. neglectus & + & - & + & - & - & + & - & + & - & + \\
\hline & Paratylenchus & $14.9 \mathrm{c}$ & $22.3_{b}$ & $65.2 \mathrm{a}$ & $33.8_{b}$ & $26.4_{b}$ & $10.4_{\mathrm{C}}$ & $0_{\mathrm{d}}$ & $55.5 \mathrm{ab}$ & $3.75 \mathrm{~d}$ & $50 \mathrm{ab}$ \\
\hline & Tylenchus & $2.55 \mathrm{~d}$ & $25.6_{b}$ & $30.7_{b}$ & $26.3_{b}$ & $0 \mathrm{e}$ & $8.5 \mathrm{c}$ & $15.77_{c}$ & $90.5 \mathrm{a}$ & $38.5_{b}$ & $15_{c}$ \\
\hline & Psilenchus & $10_{b}$ & $15_{b}$ & $25.3 \mathrm{a}$ & $11_{b}$ & $1.5_{\mathrm{c}}$ & $10_{b}$ & $0_{\mathrm{d}}$ & $0_{\mathrm{d}}$ & $0 \mathrm{~d}$ & $2.5_{\mathrm{c}}$ \\
\hline & Tylenchorhynchus & $82.3 \mathrm{a}$ & $33.8 \mathrm{~cd}$ & $15.5 \mathrm{~d}$ & $8.6 \mathrm{~d}$ & $24.3 \mathrm{~cd}$ & $31.5 \mathrm{~cd}$ & $45.5_{c}$ & $26.3 \mathrm{~cd}$ & $60_{b}$ & $43.9 \mathrm{bc}$ \\
\hline \multirow{8}{*}{ 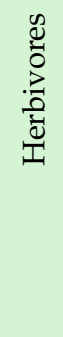 } & Merlinius & $9.77_{b}$ & $23.1 \mathrm{a}$ & $21.1 \mathrm{a}$ & $3.75_{c}$ & $4.25_{c}$ & $10_{\mathrm{b}}$ & $4.9 \mathrm{c}$ & $0_{\mathrm{d}}$ & $11.3_{\mathrm{b}}$ & $0_{\mathrm{d}}$ \\
\hline & Longidorus & $0_{\mathrm{c}}$ & $15 \mathrm{a}$ & $0_{c}$ & $7.5_{b}$ & $12.3 \mathrm{a}$ & $15.1 \mathrm{a}$ & $0_{c}$ & $0_{c}$ & $0_{\mathrm{c}}$ & $0_{c}$ \\
\hline & Heterodera & $97.5 \mathrm{a}$ & $30.6 \mathrm{~d}$ & $105 \mathrm{a}$ & $17.2 \mathrm{e}$ & $42_{c}$ & $13.2 \mathrm{e}$ & $36 \mathrm{~d}$ & $75.88_{b}$ & $46.3_{c}$ & $24.3 \mathrm{de}$ \\
\hline & H. avenae & + & - & + & + & + & - & + & + & + & + \\
\hline & H. latipons & + & + & + & - & + & + & - & + & + & - \\
\hline & Hoplolaimus & $120 \mathrm{a}$ & $5.3_{c}$ & $60_{b}$ & $0_{\mathrm{d}}$ & $0_{\mathrm{d}}$ & $6.2 \mathrm{c}$ & $9.75_{c}$ & $0_{\mathrm{d}}$ & $0_{\mathrm{d}}$ & $0_{\mathrm{d}}$ \\
\hline & H. indicus & + & + & + & - & - & + & + & - & - & - \\
\hline & Helicotylenchus & $105 \mathrm{a}$ & $19.6 \mathrm{~d}$ & $27.1_{\mathrm{c}}$ & $31.09 \mathrm{c}$ & $29.3_{c}$ & $25.6_{c}$ & $15.9 \mathrm{~d}$ & $35_{c}$ & $41.3_{\mathrm{b}}$ & $19.6_{\mathrm{d}}$ \\
\hline
\end{tabular}




\subsection{Diversity of Nematode Communities in Wheat}

The diversity patterns of soil nematodes were investigated through the calculation of several diversity indices (Figure 4). The maturity index (MI), which focuses on freeliving taxa was significantly different between the localities surveyed $\left(F_{\text {index }}=65.7 ; d f=9\right.$; $p<0.001$ ). The highest values were observed in Ait Melloul (2.81), Biougra (2.93), Massa (2.84), and Ouled Dahou (2.98) (Figure 4a), while both Lqliaa and Sidi Boushab provinces had the lowest MI values (1.97 and 2.11), respectively. For the plant-parasitic index (PPI), no significant differences were observed between the localities $\left(F_{\text {index }}=23.1 ; d f=9 ; p>0.05\right)$ (Figure $4 \mathrm{~b}$ ) as all sampled fields showcased PPI values of $>2.8$. The channel index (CI), which implies the decomposition aspects of both fungivorous and bacterivorous nematodes showed significant differences between wheat fields from different areas $\left(F_{\text {index }}=89.4\right.$; $d f=9 ; p<0.001$ ). Both Temsia and Ouled Dahou displayed high CI values of 63.73 and 66.8, respectively, compared to the other localities (Figure 4c). Lqliaa province had the lowest CI value (13.86). As for the structure index (SI), the corresponding areas were significantly fluctuating $\left(F_{\text {index }}=75.2 ; d f=9 ; p<0.001\right)$. Three provinces (Ait Melloul, Massa, and Ouled Dahou) showed high SI values $(74<\mathrm{SI}<78)$, whereas both Ait Amira and Sidi Boushab provinces showed low SI values of 39.4 and 38.9, respectively (Figure $4 \mathrm{~d}$ ).

(a)
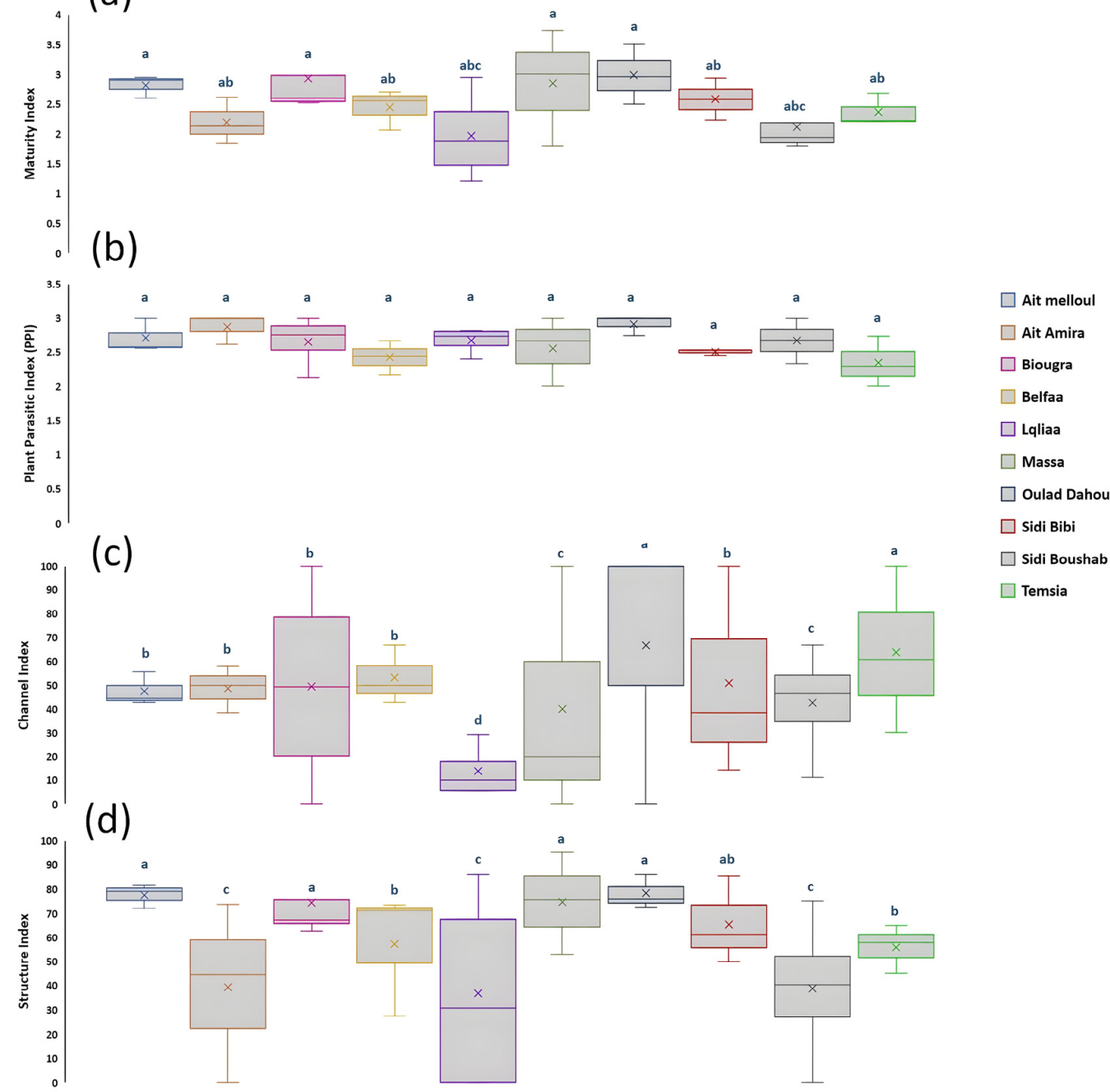

Figure 4. Nematode diversity patterns in sampled wheat fields of Southern Morocco. (a) Maturity index (MI); (b) plant-parasitic index (PPI); (c) channel index (CI); (d) structure index (SI). Letters represent homogeneous groups according to the LSD test $(p<0.05)$. 
The succession of c-p groups focusing on free-living nematodes can be an important indicator of soil status in wheat fields. For that reason, a c-p triangle was established for all nematodes obtained from different localities (Figure 5). The majority of soil samples taken from wheat fields were close to stress conditions, as c-p 3-5, which are the structure nematodes, tend to decrease drastically. On the other hand, basal nematodes (c-p 2) were significantly increased alongside the decrease of enrichment nematodes (c-p 1). Only two fields from Ait Amira and Ait Melloul localities were shown to have stable soils, while some fields from Lqliaa province had highly enriched soils.

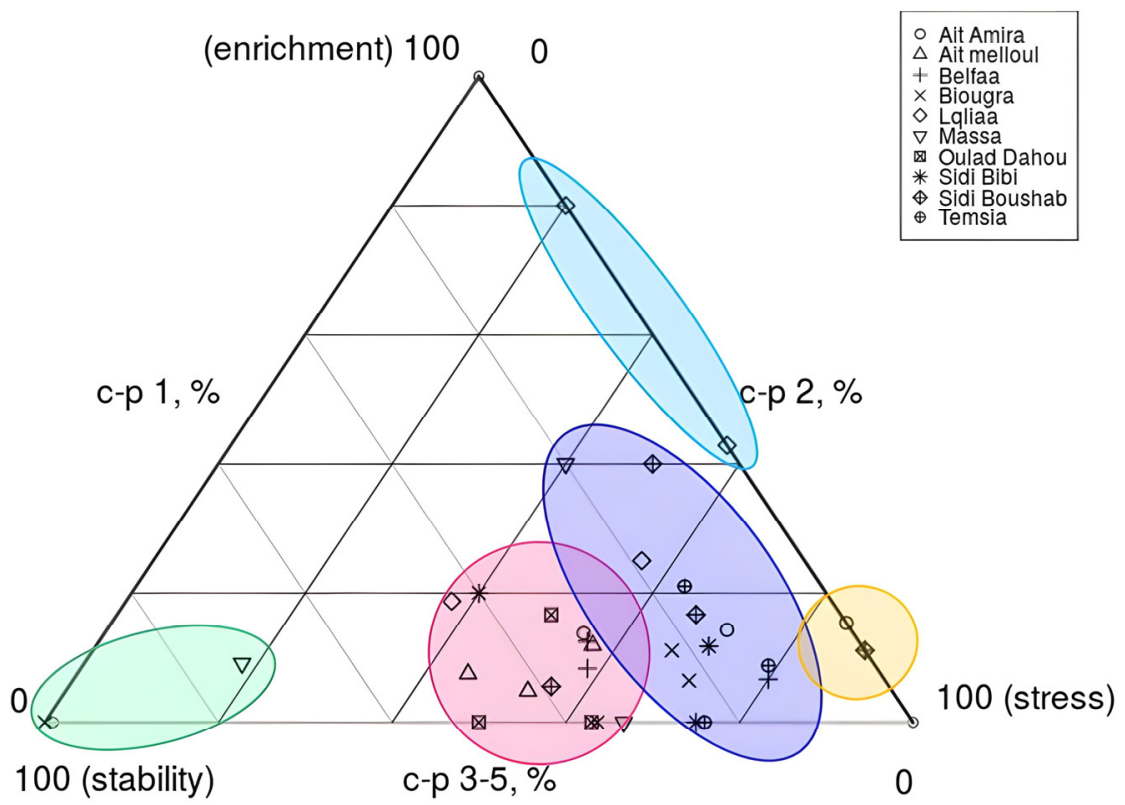

Figure 5. c-p triangle depicting soil status regarding nematodes c-p groups evolution in wheat fields surveyed.

Table 4 depicts the number of taxa, the Shannon-Weaver diversity index $\left(\mathrm{H}^{\prime}\right)$, and the evenness (J) values for each cereal growing field surveyed. The Shannon-Weaver diversity index was significantly $(p<0.05)$ higher in Oulad Dahou, Ait Melloul, and Sidi Boushab $(2.91,2.7$, and 2.49 , respectively), compared to the other localities. The lowest index value was reported in Ait Amira province (1.2). There was no significant difference in evenness between all localities.

Table 4. Diversity of nematode communities of wheat in the different surveyed localities.

\begin{tabular}{cccc}
\hline \multirow{2}{*}{ Localities (Province) } & \multicolumn{3}{c}{ Diversity Parameters } \\
\cline { 2 - 4 } & $\begin{array}{c}\text { Mean Number } \\
\text { of Taxa }\end{array}$ & $\begin{array}{c}\text { Shannon Diversity } \\
\text { Index (H') }\end{array}$ & Evenness (J) \\
\hline Ait Amira & $17.4 \mathrm{c}$ & $1.2 \mathrm{bc}$ & $0.68 \mathrm{a}$ \\
Ait Melloul & $28.56 \mathrm{a}$ & $2.7 \mathrm{a}$ & $0.8 \mathrm{a}$ \\
Belfaa & $23.4 \mathrm{ab}$ & $2.54 \mathrm{a}$ & $0.73 \mathrm{a}$ \\
Biougra & $6.27 \mathrm{~d}$ & $1.05 \mathrm{bc}$ & $0.71 \mathrm{a}$ \\
Lqliaa & $21.83 \mathrm{~b}$ & $2.2 \mathrm{ab}$ & $0.83 \mathrm{a}$ \\
Massa & $19.48 \mathrm{~b}$ & $2.17 \mathrm{ab}$ & $0.78 \mathrm{a}$ \\
Oulad Dahou & $30.7 \mathrm{a}$ & $2.91 \mathrm{a}$ & $0.89 \mathrm{a}$ \\
Sidi Bibi & $15.4 \mathrm{c}$ & $1.83 \mathrm{ab}$ & $0.87 \mathrm{a}$ \\
Sidi Boushab & $25 \mathrm{ab}$ & $2.49 \mathrm{a}$ & $0.77 \mathrm{a}$ \\
Temsia & $20.55 \mathrm{~b}$ & $1.63 \mathrm{~b}$ & $0.74 \mathrm{a}$ \\
\hline$p$ & $<0.001$ & 0.0365 & 0.795 \\
\hline
\end{tabular}

Means in the same column followed by different letters are significantly different based on Tukey's test. 


\subsection{Nematodes as Potential Soil Bio-Indicators in Wheat Fields}

Principal component analysis of the soil parameters across the surveyed cereal fields (Figure 6) showed that the fraction of variance accounted for by the first two PC axes was $26.15 \%$ and $15.32 \%$ (eigenvalues), respectively. A loading plot of the soil factors (Figure 6a) indicated that the PC1 axis was related to mineral content (mainly Mn and Zn) alongside clay content (Cla) and $\mathrm{pH}$ in negative $\mathrm{PC}$ values and calcium (Cal), sandy content (San), and iron ( $\mathrm{Fe}$ ) in positive PC values. The PC2 axis was related to the total organic matter (Tom) and humic organic matter (Hom) content, C:N ratio, and to a lesser extent to phosphorus $(\mathrm{P})$ and copper $(\mathrm{Cu})$. The significance of this relationship was assessed via an enhanced heatmap (heatmap-2) (Figure 6b). It was indicated by the analysis that all nematode taxa identified were significantly correlated with calcium, Mn content, and total organic matter (Tom). Interestingly, soil texture patterns (sand, clay, and silt content) were also positively correlated with many soil nematode taxa. For instance, Aphelenchoides spp., Prismatolaimus spp., Mononchus spp., and Pratylenchus spp. were abundant in sandy soils, whereas Dorylaimus spp., Longidorus spp., and Panagrolaimus spp. were more abundant in clay soils. Except for Merlinius spp., Panagrolaimus spp., and Eucephaloubus spp., most of the nematodes were not attracted or slightly attracted to silt content. Regarding mineral contents, few $(\mathrm{Mn}, \mathrm{K})$ were shown to have a significant positive relationship with some taxa thus enhancing their occurrence, while the rest $(\mathrm{Zn}, \mathrm{N}, \mathrm{Na}, \mathrm{P}, \mathrm{Cu}$, and $\mathrm{Fe})$ were not affiliated very closely with these nematodes. The root-knot nematode (Meloidogyne spp.), the root-lesion nematode (Pratylenchus spp.), and the spiral nematode (Helicotylenchus spp.) were strongly linked to the potassium $(\mathrm{K})$ content, while most of the taxa, excluding Paratylenchus spp., Meloidogyne spp., Eudorylaimus spp., Thornenema spp., Metateratocephalus spp. and Hoplolaimus spp., showed decent activity represented by high abundance in the presence of high calcium concentrations in soil. Electrical conductivity slightly affected two genera (Ditylenchus spp. and Pratylenchus spp.) while Tom had a positive impact on some nematode communities (Acrobeles spp., Eucephalobus spp., and Heterodera spp.).

The potential of nematodes as effective soil health bio-indicators in wheat fields was assessed using food web diagnostics (Figure 7). The food web analysis was conducted for all nematode taxa identified in all sampled localities. This analysis highlighted the soil health status of each province in the function of occurred nematode communities (Figure 7a). More than $50 \%$ of the wheat fields were shown to have mature, N-enriched, regulated soils with a bacterial feature. Eight fields representing Sidi Bibi, Oulad Dahou, Biougra, Ait Melloul, Massa, and Temsia were characterized by fertile, suppressive soils with a moderate $\mathrm{C}: \mathrm{N}$ ratio and bacterial-fungal combination propriety. On the other hand, most of the Sidi Boushab and Ait Amira fields were shown to be disturbed and conductive with a low C:N ratio. Only two field soils (from Belfaa and Sidi Boushab provinces) were presumably considered to be degraded with depleted nutrient resources and fungal nature. To further explain the latter analysis, metabolic footprint analysis was conducted in the sense all localities are clearly distinguished in terms of their soil health (Figure $7 \mathrm{~b}$ ). Generally, this analysis is based on the carbon metabolism of nematode taxa and represents the sum of their production and respiration patterns. The amplitude of structure and enrichment indices are shown by structure footprint (SMF) and enrichment footprint (EMF), respectively. Four localities (Massa, Sidi Bibi, Belfaa, and Ait Melloul) were distinctively highly enriched and matured with a high abundance of bacterial feeders. Furthermore, fertile soils were accurately presented in Oulad Dahou, Temsia, and Biougra provinces. Soil disturbance in wheat fields was highlighted mainly in Ait Amira, Laqliaa, and Sidi Boushab provinces. Despite all these observations, it was noticed that most of the localities had EMF $<<$ SMF. 
(a)

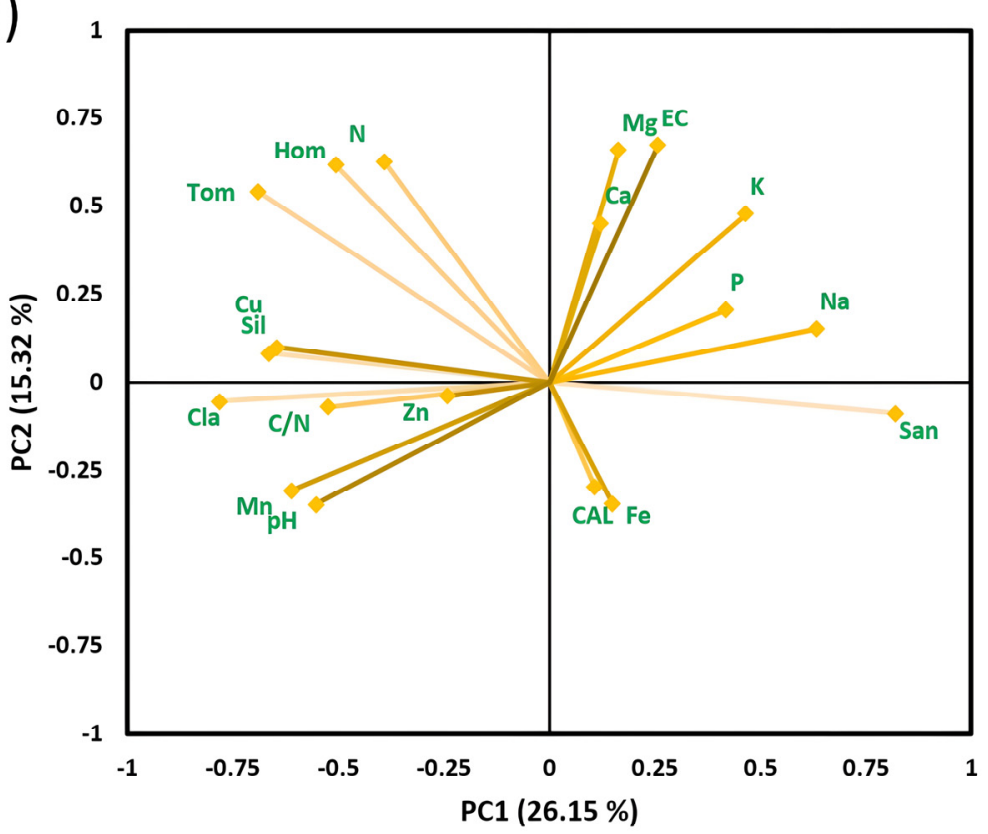

(b)
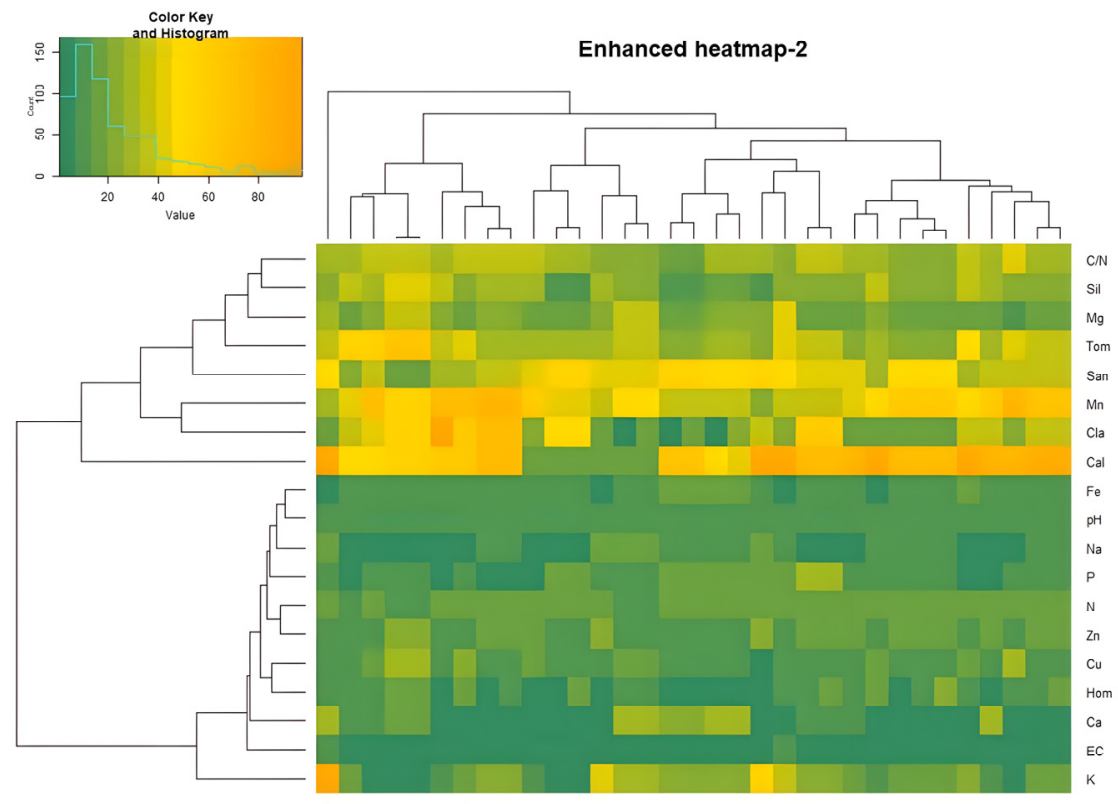

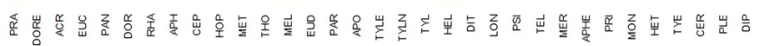

Figure 6. Physical-chemical soil patterns and correlation with nematode assemblies. (a) PCA loading plot for soil characteristics. (b) Advanced heatmap (enhanced heatmap-2) showing the population structure of nematode taxa in relationship with soil parameters. Ward's clustering algorithm was applied to the Spearman dissimilarity matrix of nematode distribution in wheat fields in interaction with physical-chemical soil characteristics. The upper dendrogram represents nematode patterns. The left dendrogram represents soil patterns. The color key scale represents the normalized Row Z-score of nematode abundance (per $100 \mathrm{~cm}^{3}$ of soil). 


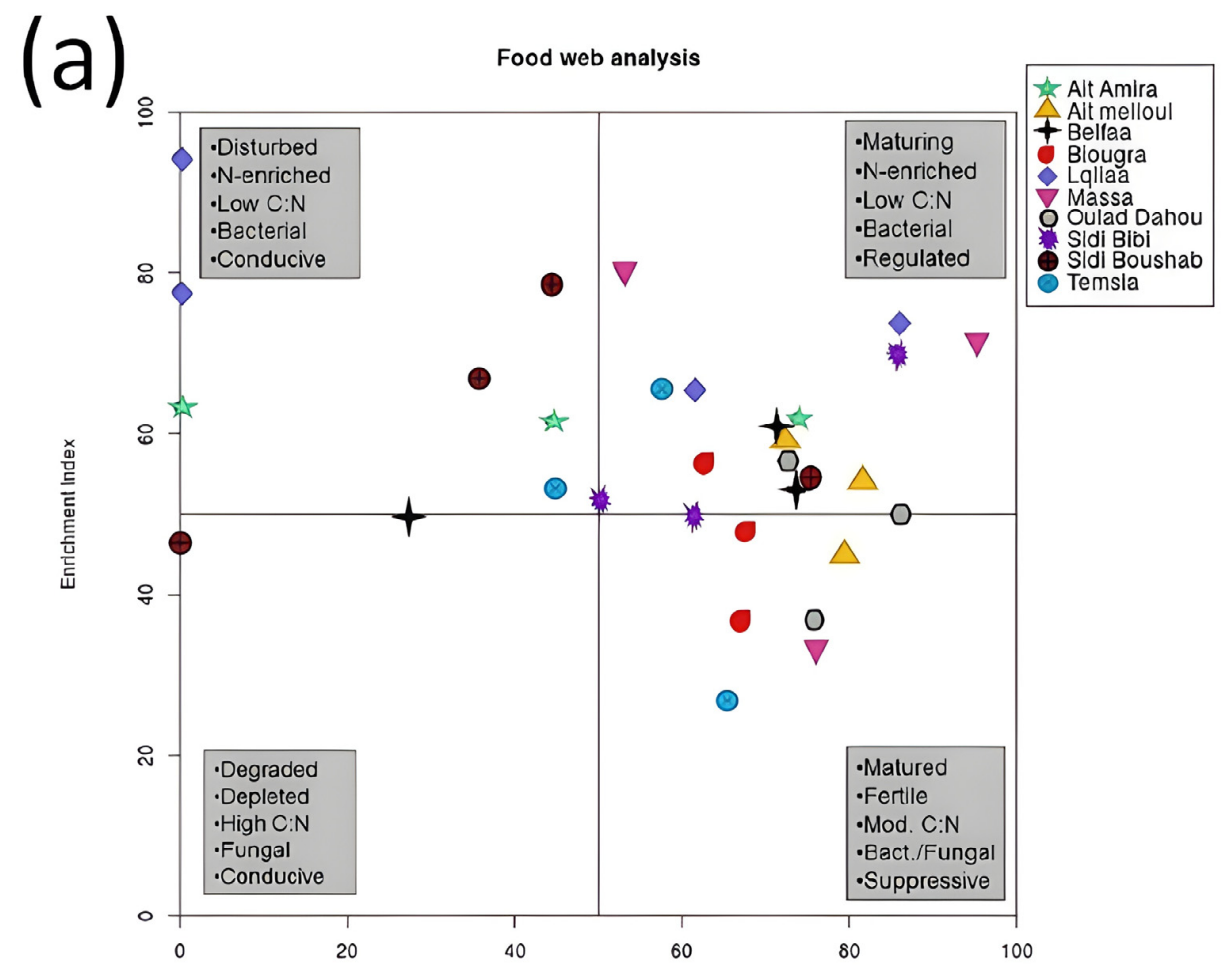

(b)

Struolure Index

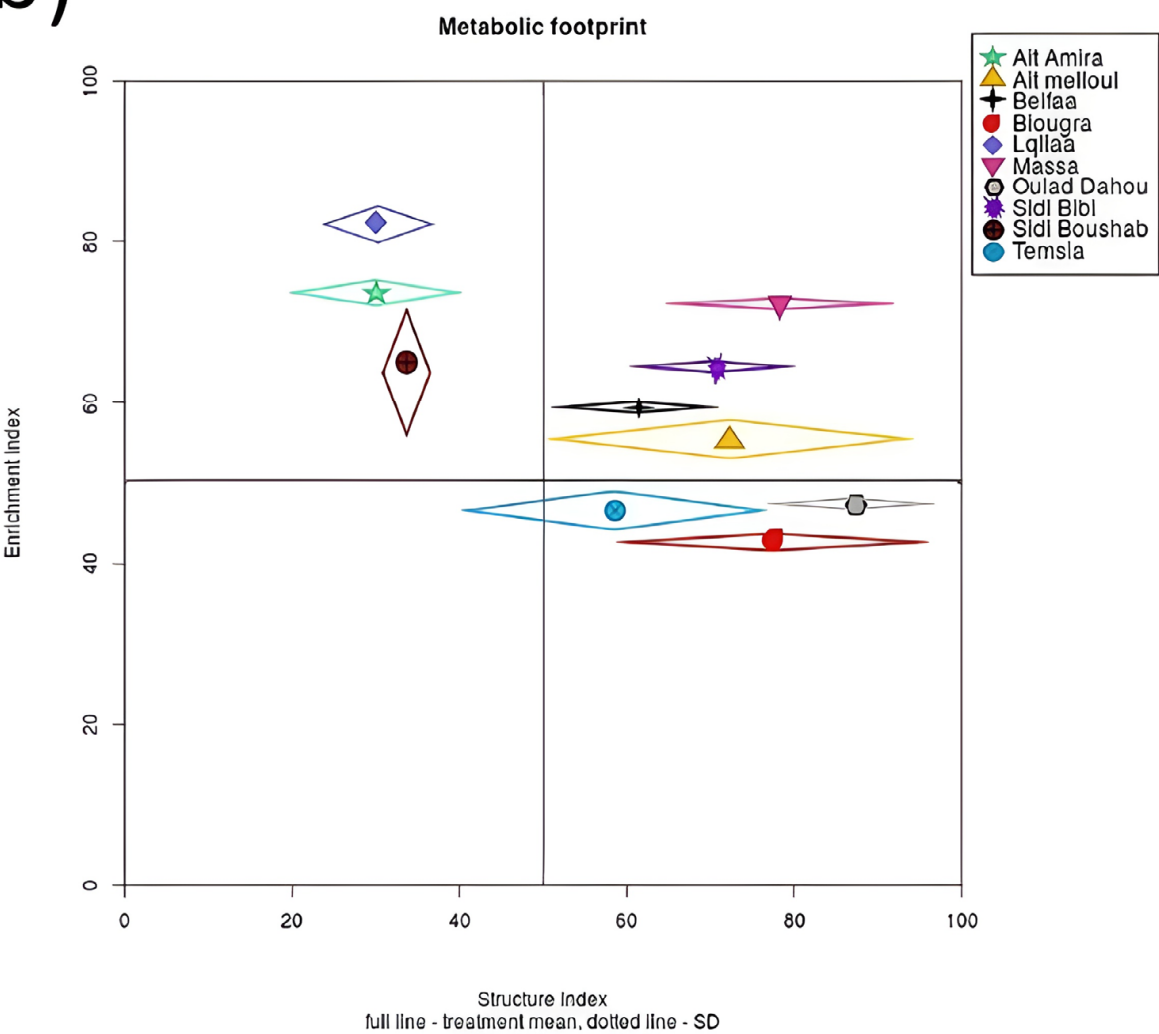

Figure 7. Nematode food web diagnostics and their position as soil health bioindicators. (a) Food web analysis of nematode communities at different wheat areas sampled. (b) Metabolic footprint showing the amplitudes of nematode enrichment and structure indices throughout wheat areas surveyed. 


\subsection{Nematode Guild Dynamics with Soil Health Attributes}

For a better understanding of soil health bioindication potential embodied within soil nematodes, spatial contribution analysis was conducted via projecting the ANOSIM and SIMPER analyses outputs. This analysis is meant to highlight the main contributors in disturbed and undisturbed wheat soils in a spatial manner (Figure 8). In undisturbed soils, plant-feeding nematodes (PF) were the most contributors with 6 taxa including Pratylenchus spp. (50\%) and Helicotylenchus spp. $(20 \%)$ as revealed by the unidimensional plot (Figure 8a). The contribution of bacterial feeders (BF) was explained by five taxa, mainly Acrobeles spp., Rhabditis spp., and Plectus spp. Therefore, fewer taxa were shown to operate in these soils, as fungal feeders (FF) (e.g., Ditylenchus spp. and Aphelenchoides spp.) and omnivorous nematodes (P) (e.g., Eudorylaimus spp. and Thornenema spp.) were only represented by three taxa. On the other hand, in disturbed localities, the unidimensional plot revealed the nematode contributors from each feeding type (Figure $8 \mathrm{~b}$ ). For instance, Rhabditis spp., Cephalobus spp., and Diplogasteriana spp. were most involved as bacterial feeders (BF). Fungal feeders (FF) were represented by Ditylenchus spp. (65\%), Aphelenchus spp. (20\%) and Aphelenchoides spp. (9\%). The root-lesion nematode (Pratylenchus spp.) has dominated the plant-feeding (PF) areas by $54 \%$ followed by Helicotylenchus spp. $(23 \%)$ and Paratylenchus spp. (10\%). Moreover, omnivores were contributed via Aporcelaimus spp. $(80 \%)$ and Dorylaimus spp. $(15 \%)$, while predatory nematodes were only contributed via Mononchus spp. (100\%). The multidimensional bubble plot has shown that all nematode feeding groups were spatially distributed in a hierarchic mode based on their relative abundance and occurrence. In low occurrence values, bacterial and fungal feeders were highly abundant with high contribution (90-100\%) to the soil. This abundance showed different aspects (moderate to low) depending on the frequency occupied by nematodes. Plant feeders and omnivores contributed mostly to moderate frequencies. However, they extend their roles in extreme conditions where all taxa can exist altogether. Overall, the soil disturbance has revealed better nematode taxa contribution compared to undisturbed soils.

The succession of nematode functional guilds (FGs) and trophic levels (TLs) was channeled through a machine learning-related analysis for a better understanding of its affiliation with soil disturbance attributes observed throughout the surveyed wheat regions (Figure 9). The Venn diagram depicts the possible combinations that might occur in soils (Figure 9a). Successions started with plant feeders (PFs) were shown to be consistently associated with fungal feeders (FFs) (WSRP $=60 \% ; p<0.001$ ) compared to other TLs linkages. Furthermore, bacterial feeders (BFs) had prominent relationship with omnivore organisms (Os) (WSRP $=50 \% ; p<0.001)$. Tripartite successions were not much anticipated in the sense that low WSRP values were recorded. On the other hand, 30\% of all FGs (PF-FF$\mathrm{BF}-\mathrm{O}-\mathrm{P}$ ) have occurred in the same frame (soil or rhizosphere platforms). Functional guilds ${ }^{\prime}$ performance was visualized through a lollipop plot (Figure 9b). In terms of disturbance status, PFs and FFs were performing better under disturbed conditions while BFs prevailed in undisturbed soils. On top of that, a balanced profile was predicted via projecting nematode data of both disturbed and undisturbed soils to the machine learning model algorithm incorporated into lollipop plot libraries. This profile had relatively moderate to high performance of PFs, BFs, and Os, alongside moderate to low FFs and Ps which could provide optimal conditions for soil health growing wheat crops. 
(a)

(b)
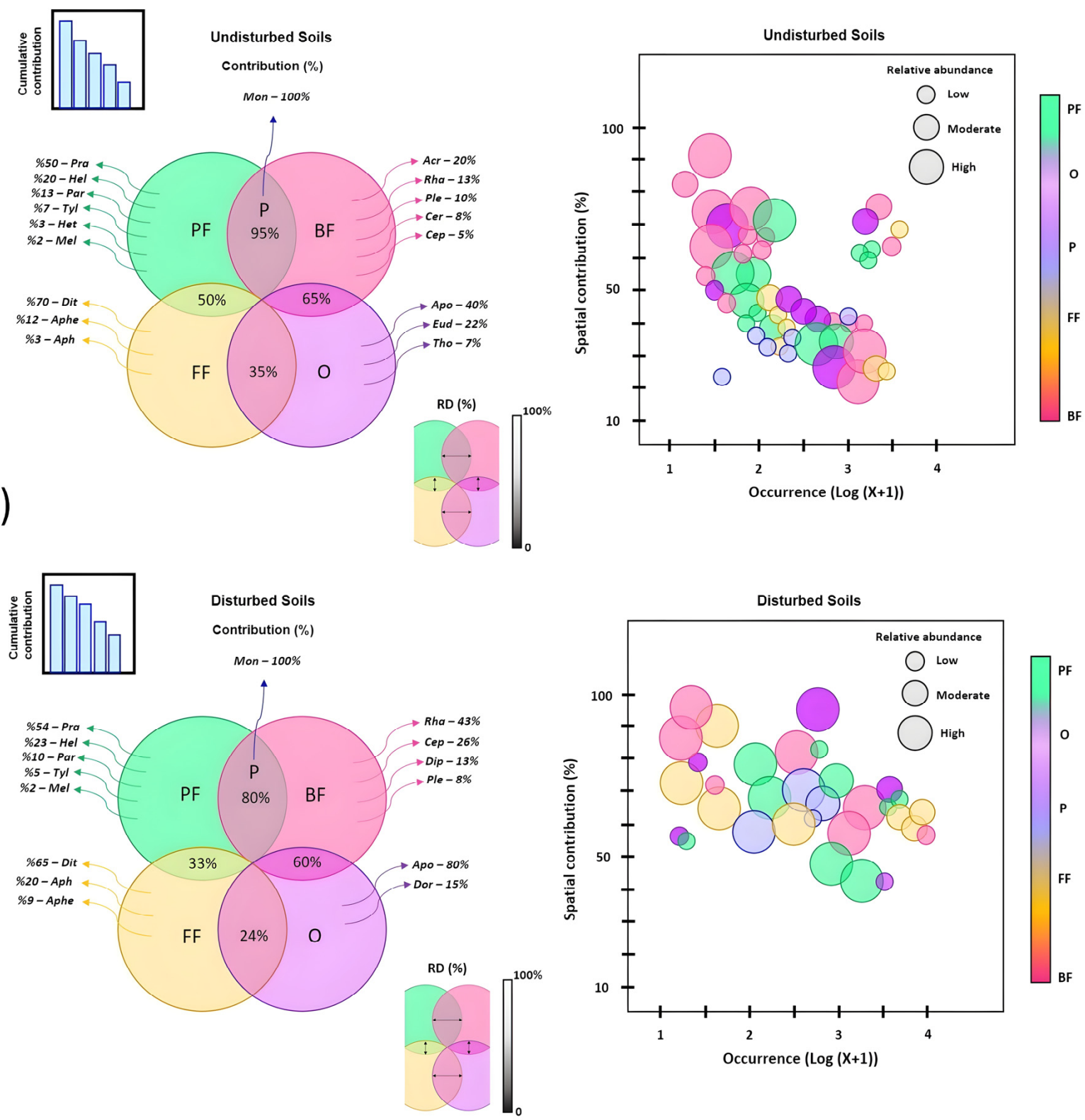

Figure 8. Nematode contribution patterns to agricultural wheat soil health. (a) Spatial contribution percentage of all soil nematode feeding types in undisturbed cereal soils. (b) Spatial contribution percentage of all soil nematode feeding types in disturbed cereal soils. The unidimensional plot represents the projected contribution values according to the ANOSIM and SIMPER analyses. These values were plotted in a multidimensional spatial bubble plot using a predicted model based on nematode abundance in each contribution pattern. Relative distance (RD) was calculated and computed between nematode trophic groups. The plots were generated using BioPython matplotlib library via Yellowbrick machine learning module [55] and sviewgui PyQt-based GUI packages [56] in PyCharm API. 
(a)
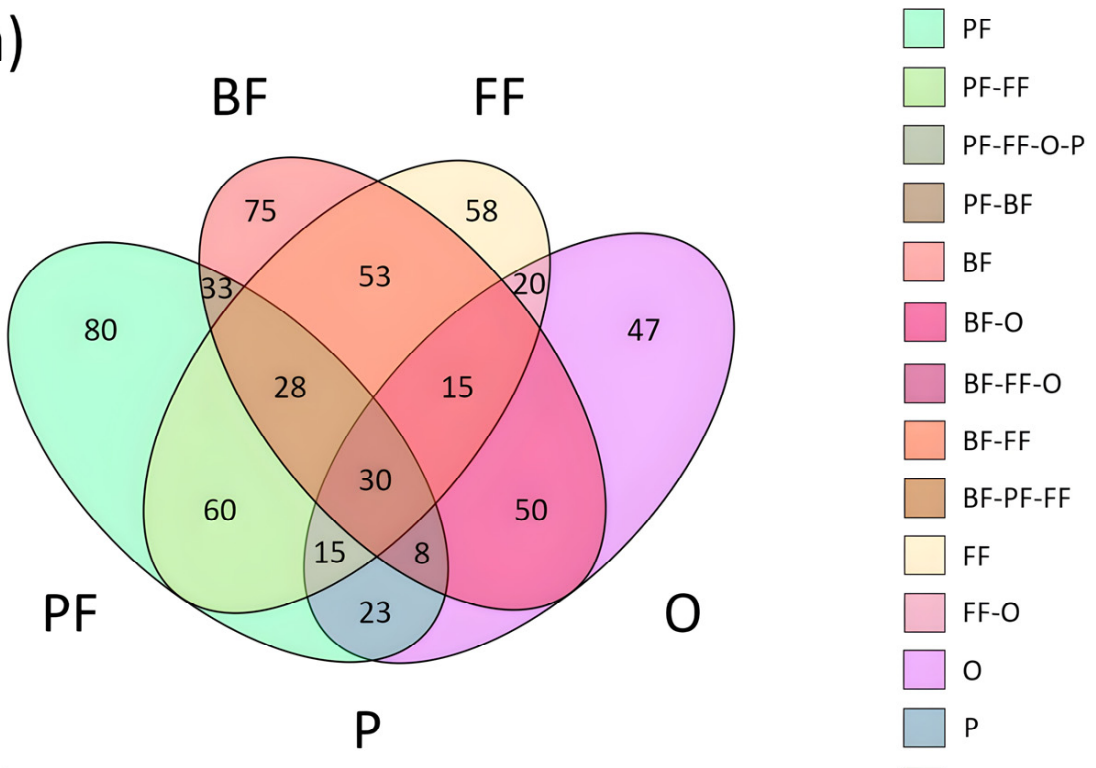

(b)
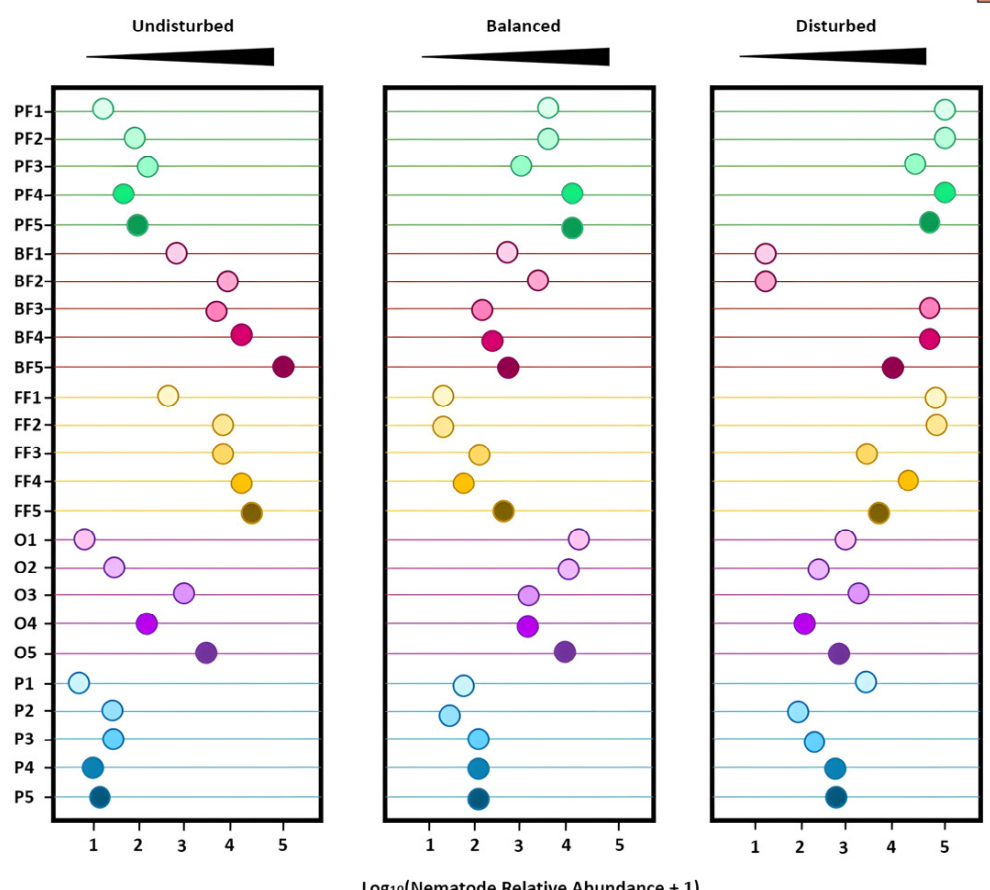

P-BF-O

PF-FF-BF-O-P

$\log _{10}($ Nematode Relative Abundance +1 )

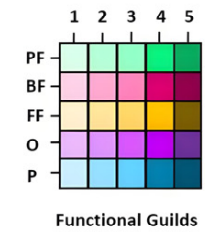

Figure 9. Functional guilds and trophic levels succession of soil nematodes on soil disturbance attributes in wheat fields. (a) Venn diagram depicting all nematode trophic levels successions. The numbers represent the weighted species richness percentage (WSRP) calculated in all surveyed areas. (b) Lollipop plot of nematode functional guilds (FGs) performance patterns for undisturbed, balanced, and disturbed soils. FGs were cube-space $(5 \times 5)$ organized and classified according to their trophic position (1 to 5 scaling). The plots were generated using BioPython matplotlib library via Matplotlib-venn [59] and Matplotlib-lollipop [60] modules in PyCharm API. 
The potential of the nematode community as soil bioindicators has proven its association with soil health through their effective spatial contribution and abundance. These parameters were projected and clustered via a parsimony mapping network (PMN) analysis to showcase the distinct association strength between cereal soil types and nematode feeding types (with some of their corresponding species) for better soil health understanding linking nematodes diversity as a key factor (Figure 10). In clay soils, the parsimony association was strong with three feeding types, providing those bacterial feeders had closer distance $\left(E d_{B F}=3.4 ; 96 \%\right.$ linkage $)$ followed by fungal feeders $\left(E d_{F F}=7.1 ; 45 \%\right.$ linkage $)$ and plant feeders $\left(E d_{B F}=7.9 ; 70 \%\right.$ linkage $)$. However, the latter is shown to have more diverse attributes. On the other hand, omnivorous nematodes were normally more associated with silty soils $\left(\mathrm{Ed}_{\mathrm{O}}=3.9 ; 70 \%\right.$ linkage $)$ with high weighted abundance. Fungal feeders were strongly related to the moderate abundance of Ditylenchus spp. presumably D. dipsaci $\left(E d_{F F}=6.1 ; 66 \%\right.$ linkage $)$. Significant parsimony values were observed in sandy soils in the sense that more nematode diversity could be observed. Plant-parasitic nematodes were more diverse with 6 taxa associated and strongly linked ( $E d_{P F}=5.3 ; 95 \%$ linkage). The root-knot nematode (Meloidogyne spp.) was predicted to be abundant in all respective species identified ( $M$. incognita, $M$. javanica, and $M$. arenaria), while the cereal cyst nematode (Heterodera spp.) was shown to contribute with both $H$. avenae and H. latipons. Besides predators $\left(E d_{P}=3 ; 27 \%\right.$ linkage), omnivores (Thornenema spp. and Aporcelaimus spp.) were likely more close to this soil matrix $\left(E d_{O}=3.7 ; 33 \%\right.$ linkage $)$ although weak parsimony association was predicted for Monochus spp. Correlations between the sampled cereal soils were also implemented, as a significant correlation was found between clayish and silty soils $\left(\mathrm{R}^{2}=0.85, p<0.001\right)$ while sandy soils were not prominently affiliated with the rest $\left(\mathrm{R}^{2}=0.45-0.56, p>0.05\right)$.

\subsection{Effect of Climatic Factors on Nematode Trophic Patterns}

Redundancy analysis (RDA) was conducted to evaluate the relationship between nematode trophic levels represented by several taxa identified and climatic/geographic factors attributed as MAP (mean annual precipitation), MAT (mean annual temperature), LA (latitude), and LG (longitude) (Figure 11). Across all localities studied, multiple correlations were detected. For instance, plant parasites (PP) were significantly related to the MAP patterns $(p<0.01)$, while bacterivore and fungivore nematodes were mostly dependent on MAT ( $p<0.01$ and $p<0.05$, respectively). Moreover, geographical attributes (latitude and longitude) were predicted to be affecting omnivores and predators, respectively. 


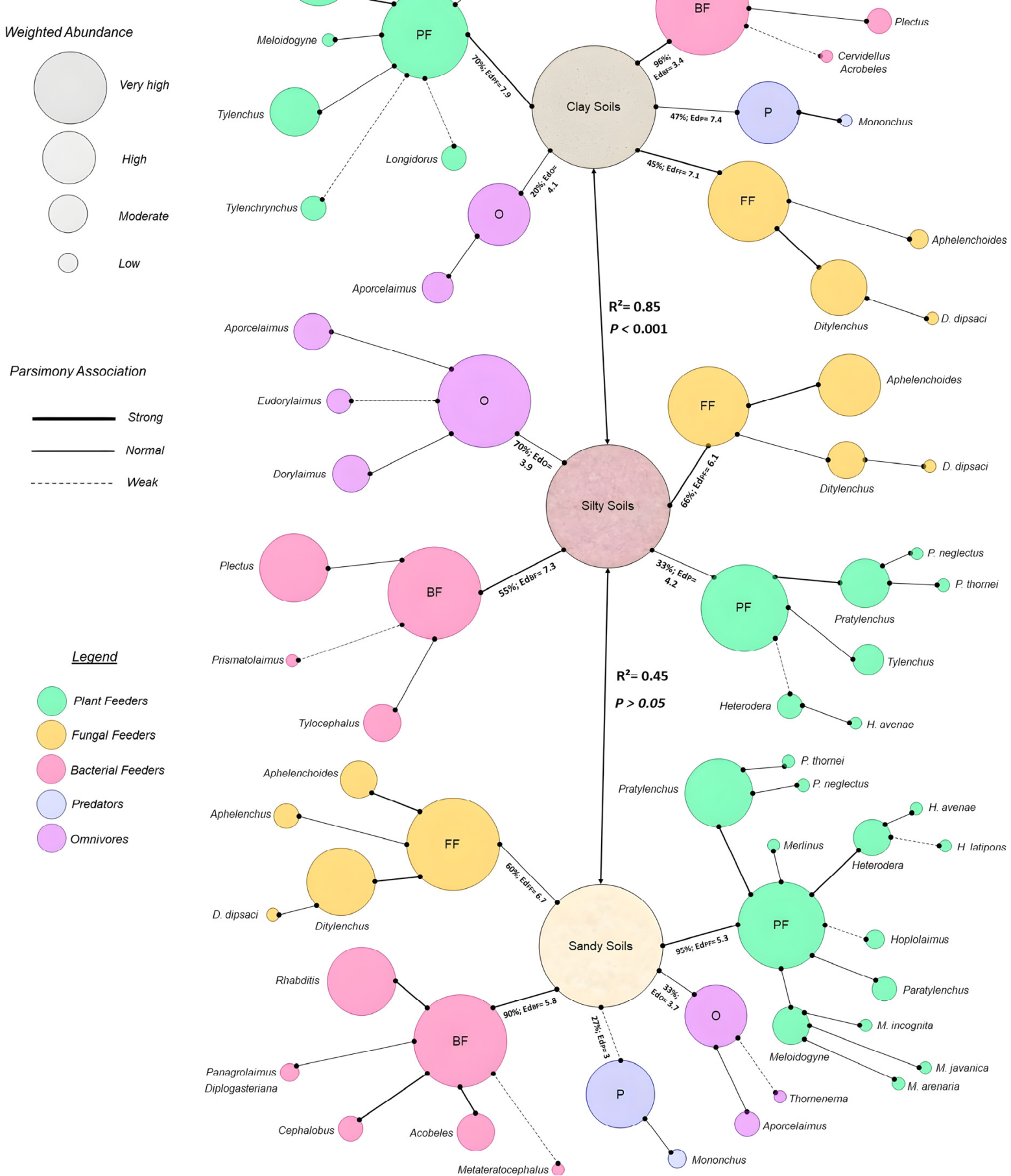

Figure 10. Parsimony mapping network (PMN) shows the association between soil type and nematode feeding types. Contribution values from multidimensional spatial bubble plots were projected and clustered for different soil patterns. The associated taxa and their affiliation strength were predicted using the Euclidean distance based on a dissimilarity matrix between all localities with similar soil patterns and linkage percentages. Weighted abundance was computed for each feeding type. The plot was generated using BioPython matplotlib library with Bio.Phylo module [57] and NetworkX library [58] in PyCharm API. 


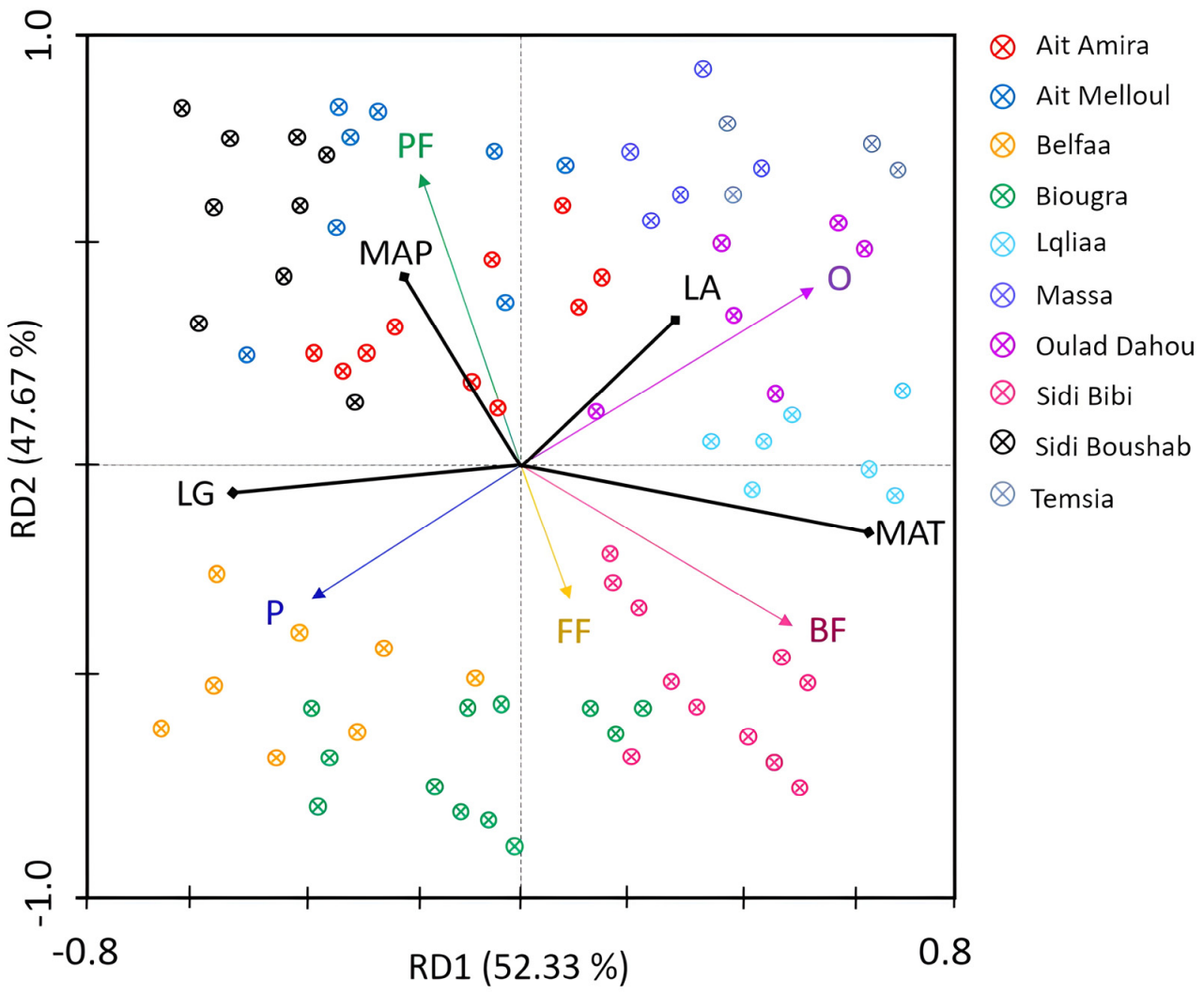

Figure 11. Redundancy analysis (RDA) of soil nematode taxa and environmental factor variables (LA-latitude; LG-longitude; MAP—-mean annual precipitation; MAT—mean annual temperature). Soil nematodes are grouped into 4 trophic guilds (BF - bacterial feeders, FF-fungal feeders, $\mathrm{PF}$ plant feeders, $\mathrm{O}$-omnivores, and $\mathrm{P}$-predators); Crossed circles indicate sampling localities; the bold black lines indicate environmental factors. The plot was generated by Python matplotlib and ordination libraries (skbio.stats.ordination) [61].

\section{Discussion}

Soil nematodes, as one of the soil's key organisms, have been considered as bioindicators to monitor soil health and quality due to the inevitable relationship between soil organisms and their environmental factors [62]. Our study provides new insights about cereal soil bio-indication through the identification of soil nematodes embodied with edaphic and climatic patterns. A total of 33 nematode taxa were founded across 80 fields from 10 different localities in the Moroccan Souss-Massa region. These genera were classified into five distinct trophic levels (bacterivores, fungivores, omnivores, predators, and herbivores). Generally, most of the scientific research related to plant nematology in cereals is strictly focused on PPNs, neglecting the occurrence and biodiversity of the free-living ones. The concept of soil health and quality relies more on bacterial-fungal decomposition channels governed by beneficial organisms [63]. Yildiz et al. [64] detected 43 nematode genera across two cereal-growing regions in Turkey (i.e., 12 bacterivores, 4 fungivores, 10 omnivores, 4 predators, and 13 herbivores). While Liu et al. [65] identified 24 genera associated with jujube (Ziziphus jujuba M.)/ wheat (Triticum aestivum L.) intercropping systems in the Xinjiang arid region, northwest of China (i.e., 12 bacterivores, 4 fungivores, 1 omnivore, and 7 herbivores).

Bacterivorous nematodes were distributed in 7 families with the predominance of Cephalobidae and Rhabditidae families. In addition, the c-p 2 functional guilds were the most abundant among all. This is similar to the results obtained by Liu et al. [65], as they stated that Acrobeloides genera belonging to the Cephalobidae family have a very decent abundance alongside the dominance of the c-p 2 functional guild. Moreover, Diakhaté [66] indicated that Piliostigma reticulatum, a shrub from the Fabaceae family in cereal fields, can 
promote the proliferation of bacterivorous nematodes (especially from the Cephalobidae family). This family was reported to be the most abundant in soils [67]. Aphelenchoides, Aphelenchus, and Ditylenchus genera belonging to Aphelenchoididae, Aphelenchidae, and Anguinidae families, respectively, were more frequent throughout all localities surveyed. Song et al. [68] reported the same finding in wheat soils, with a high prevalence of Aphelenchus spp. Fungivore nematodes have significant importance in diversified soils and cropping systems [69]. Furthermore, the ratio of bacterivore/fungivore is known to be a reliable indicator of organic matter decomposition in soil [70]. Omnivores have minimalistic proportions in all prospected fields. This can be explained by the fact that the first $15 \mathrm{~cm}$ of soil has low levels of this group, so they can probably impact populations of other nematode groups [71]. These nematodes are relatively large and more sensitive to environmental constraints [72].

Regarding plant-parasitic nematodes, 11 genera were identified in our study. Specifically, the root-lesion nematodes (Pratylenchus spp.), cereal cyst nematodes (Heterodera spp.), and root hair feeders (Tylenchus spp.) were the most prevalent across cereal-growing fields surveyed. Mokrini et al. [20] reported the same finding via identifying the same nematode genera in several Moroccan regions, as they are strongly associated with wheat. In addition, Lui et al. [65] mentioned that Tylenchus spp., belonging to the Tylenchidae family with the c-p 2 functional guild, was more abundant in wheat intercropping systems. Tylenchorynchus spp. has strong distribution in most localities. Ogolla [73] found high frequencies of this nematode in maize fields alongside Pratylenchus spp. On the other hand, three RKNs species were detected including $M$. incognita, $M$, javanica, and $M$. arenaria. Their abundance was moderately presented compared to the other important PPNs due to their inconsistencies with cereal crops. Moreover, previous reports supported the idea of having other RKN species in highly infested wheat fields such as M. artiellia [6]. However, since this species requires optimal edaphic conditions to occur [6], it is inevitable for our survey not to detect it as the temperatures in southern Morocco are relatively high for extensive periods.

Measures of the ecological succession of nematode communities include the fungivore to bacterivore ratio $(\mathrm{Ba} / \mathrm{Fu})$, maturity (MI, MI2-5), channel (CI), and structure (SI) indices $[22,24]$. The fungivore to bacterivore ratio indicates the successional stage of the decomposition aspect. The nematode maturity index (MI) [22] is focused on the sensitivity of different taxa to the stress pattern, and it is scaled from 1 to 5 values. These indices measure the degree of disturbance that occurred in a certain ecosystem. This disturbance can interrupt the ecological succession of a soil community and hence its history can be distinguished [74]. Freckman and Ettema [75] highlighted that both the maturity index $(\mathrm{MI})$ and plant-parasitic index $(\mathrm{PPI})(2 \leq \mathrm{PPI} \leq 5)$ are particularly used to study the effects of disturbances on nematode communities in agroecosystems. Low values indicate high disturbance alongside the important presence of colonizers. On the other hand, increased values define low disturbance patterns with a high degree of persistent nematodes [26]. In our study, cereal fields of Lqliaa and Sidi Boushab had low MI values compared to the rest. Regarding the channel index (CI), differences were observed between prospected localities providing that all decomposition channels of organic matter were different depending on the abundance of fungivores and bacterivores. Ruess and Ferris [76] mentioned that disturbed systems have decomposition aspects dominated essentially by fungi organisms. Therefore, fungi decomposition is considered to be slow compared to the bacteria counterpart. Diversity has been related to the numbers of nematode taxa, and it seems that there is a misconception about it. Rather, diversity incorporates numbers of nematode genera (i.e., richness $(S)$ ) and equitability (i.e., evenness $(\mathrm{J})$ ). Even though Shannon $\left(\mathrm{H}^{\prime}\right)$ [49] and Simpson $(\lambda)$ [77] indices are the most familiar, there are several diversity indices, each having relative potential attributes. Generally, the $\mathrm{H}^{\prime}$ index is more sensitive to rare taxa, and the $\lambda$ index considers taxa in common.

To understand the correlation between soil characteristics and soil nematodes, it is extremely important to investigate their biodiversity [78]. Nematode competition (e.g., competition over space) can be directly or indirectly influenced by soil and it has an 
important role in the nematode life cycle [79]. Our study indicated a strong relationship between some soil proprieties and nematode distribution. It was shown that soil texture (mainly sandy and clay content) affected nematode occurrence. A recent study conducted by Krif et al. [80] reported that many PPNs populations were strongly correlated with soil clay, sand, and silt content in vegetable crops, while Van Diepeningen et al. [81] demonstrated the same effect in both conventional and organic agricultural settings, and they found a prominent impact. In addition, this correlation could strongly define PPNs population structures $[82,83]$. The effect of sand texture on soil nematode occurrence on cereal crops is probably due to its irreversible linkage with soil $\mathrm{pH}$ and temperature. Sandy soils have considerably higher thermal conductivity than clay soils, which may be the cue behind their affiliation with nematode patterns. Prot and Van Gundy [84] found that sandy soils seemed to promote habitat conditions for soil nematodes, as it can enhance their mobility aspect and increase their reproduction rate [85]. Regarding the soil mineral aspect, several parameters were not significantly correlated with the nematode set. Excluding six nematodes (e.g., Hoplolaimus, Metateratocephalus, and Paratylenchus), all nematodes were significantly correlated with the calcium content. In the same manner, Mokrini et al. [86] have reported a decent linkage between nematodes and soil mineral contents (i.e., $\mathrm{Ca}, \mathrm{K}, \mathrm{Mn}$, and $\mathrm{Zn}$ ) in saffron. On the other hand, Georgieva et al. [87] indicated that nematode community structure seemed to be negatively affected by soil mineral contents, decreasing both richness and maturity indices of soil nematodes (e.g., omnivores and predators). According to our findings, nitrogen does not produce a relevant effect (attractive or depletive) on nematodes. However, Oteifa [88] showed that the population of $M$. incognita was drastically reduced in beans crops after nitrogen input. The soil organic matter (total and humic) was shown to significantly reduce the structuring of soil nematodes and decrease their vital attributes. Except for bacterivorous and fungivore nematodes, the reproduction rate of nematodes could be accurately affected by the soil organic matter [89], which can probably explain the significant decrease in some nematode taxa observed in surveyed cereal-growing systems. Similarly, Mokrini et al. [86] underlined that organic matter reduced some plant-parasitic nematode abundance in saffron. It is known that organic matter accumulation in soils could cause a decline in nematode diversity $[90,91]$. Contrarily, soil organic matter contents could be positively linked with soil nematodes [92], probably due to the presence of microbial communities (bacteria and fungi), which could significantly promote nematode occurrence and influence plant growth and development $[93,94]$. The carbon to nitrogen ratio $\mathrm{C} / \mathrm{N}$, which monitors the input of carbon and nitrogen in agricultural soils, was moderately linked to nematode patterns. High organic carbon rates were reported to have a significant relationship with nematodes biodiversity outputs [63]. These results were identical to ours as most of the nematode taxa obtained were significantly occurrent and diverse in soils with high carbon rates.

As for soil health for different cereal-growing localities in the Souss-Massa region, most of the soils were mature, with high fertility and enrichment, low to moderate $\mathrm{C} / \mathrm{N}$ ratio, bacterial/fungal decomposition channels, and regulative/suppressive characteristics. Only three localities (e.g., Ait Amira, Lqliaa, and Sidi Boushab) were shown to be disturbed or degraded, with conductive proprieties. The metabolic footprints obtained were based on enrichment and structure aspects. The enrichment footprint (EMF) is known to be responsive to resource enrichment (only opportunistic nematodes), while the structure footprint shows the importance of regulatory functions (taxa at higher trophic levels) and can illustrate non-nematode taxa with similar functions [95]. Our study showed that most soils displayed an EMF >> SMF which is unusual. However, this could be ecologically explained by the fact that there is no metabolic balance manifested in nematode taxa, or it could be that other prey were available for the predators [95]. In addition, the domination of juveniles over predator populations [95] could potentially be the ultimate reason, as many of them were accurately observed in our study.

Climatic or geographic factors can be a crucial indicator of many plant-related organisms' distribution, such as nematodes [78]. Our study evaluated the occurrence of 
soil nematodes associated with wheat with three distinct climatic factors: relative soil humidity, minimum temperature, and maximum temperature. Relative soil moisture was significantly associated with nematode abundance, but the temperature did not affect their abundance. However, the effects of temperature on nematode abundance may have been masked by other environmental factors. Nielsen et al. [78] reported that the rise of nematode abundance might be due to an increase in annual temperatures and Imren et al. [96] describe a positive correlation between $P$. thornei population densities on wheat and soil temperature. Several other studies have postulated that temperatures greater than $25{ }^{\circ} \mathrm{C}$ can increase the development and multiplication rates of Meloidogyne spp. [97], Rotylenchus spp., Xiphinema spp., Hemicycliophora spp. [98], and Pratylenchus spp. [99]. This temperature increase may also lead to an increase in fungal pathogens [100] which would further reduce wheat yield due to the formation of fungi-nematode disease complexes. Precipitation could also have a significant effect on nematode communities. Multiple studies have reported that the nematode multiplication rate is positively affected by precipitation [101,102]. This indicates that minor changes in environmental factors may have several direct and indirect consequences, including modification of nematode communities and soil properties.

\section{Conclusions}

To sum up, this study provides new insights into genera diversity, incidence, and distribution of soil nematodes associated with the wheat crop in southern Morocco, with their contribution as potential bio-indicators for soil health aspects. Nematodes from different trophic groups were differently distributed in all localities surveyed. Herbivorous taxa dominated by Pratylenchidae and Heteroderidae families alongside bacterivores represented mainly by Rhabditidae and Cephalobidae families were the most pronounced. Soils from Ait Melloul, Belfaa, Oulad Dahou, and Sidi Boushab were more diversified. On the other hand, few localities (e.g., Ait Amira and Lqliaa) were shown to be disturbed. Therefore, an equilibrium between taxa was observed in all surveyed areas. Possible soil quality patterns were thoroughly analyzed, and soil nematodes proved to be promising indicators. To our knowledge, the explicit spatial relationship of all nematode taxa from different functional guilds predicted by multidimensional contribution analysis is the first to be conducted and will add a needed finding to parasitic-saprophytic interactions. In addition, the soil networking embodied within microorganisms puts nematodes as the main actors for many biodiversity-related patterns. Due to climate change, the within soil nematode communities tend to fluctuate due to many factors (temperature and precipitation), which significantly affect soil health. More research is required to expand survey areas in all Moroccan cereal growing areas to identify all nematode taxa at the species level and study the possible nematode communities' responses to agricultural practices and environmental constraints for a better understanding of their role as soil health bioindicators. This will be helpful to farmers to establish adequate management and cereal soil processing strategies against potentially related pests.

Author Contributions: Conceptualization, S.-E.L., F.M. and A.A.D.; methodology, S.-E.L., F.M., R.L and A.A.D.; software, S.-E.L.; validation, S.-E.L., F.M., R.L., T.W., T.P. and A.A.D.; formal analysis, S.-E.L., F.M. and A.A.D.; investigation, S.-E.L., F.M. and A.A.D.; resources, R.L., T.W. and T.P.; data curation, S.-E.L.; writing—original draft preparation, S.-E.L., F.M. and A.A.D.; writing-review and editing, S.-E.L., F.M., R.L., T.W., T.P. and A.A.D.; visualization, R.L., T.W. and T.P.; supervision, F.M. and A.A.D.; project administration, F.M. and A.A.D.; funding acquisition, F.M. and A.A.D. All authors have read and agreed to the published version of the manuscript.

Funding: This research was financially supported by the National Institute of Agricultural Research (INRA).

Institutional Review Board Statement: Not applicable.

Data Availability Statement: Not applicable. 
Acknowledgments: We are grateful to the farmers for helping us by providing the necessary information during the survey.

Conflicts of Interest: The authors declare no conflict of interest.

\section{References}

1. Food and Agriculture Organization Corporate Statistical Database (FAOSTAT). New Food Balances. 2020. Available online: http:/ / www.fao.org/faostat/en/\#data/FBS (accessed on 16 May 2020).

2. Jlibene, M.; Nsarellah, N. Wheat breeding in Morocco, a historical perspective. World Wheat Book 2011, 2, 425-442.

3. Barakat, F.; Handoufe, A. Agroclimatic assessment of agricultural drought in Morocco. Sci. Chang. Planétaires Sécheresse 1998, 9 , 201.

4. Nicol, J.M.; Rivoal, R. Global knowledge and its application for the integrated control and management of nematodes on wheat. In Integrated Management ad Biocontrol of Vegetable and Grain Crops Nematodes; Ciancio, A., Mukerji, K.G., Eds.; Springer Academic Publishing: Dordrecht, The Netherlands, 2008; pp. 243-287.

5. Dababat, A.A.; Imren, M.; Erginbas-Orakci, G.; Ashrafi, S.; Yavuzaslanoglu, E.; Toktay, H.; Pariyar, S.R.; Elekcioglu, H.I.; Morgounov, A.; Mekete, T. The importance and management strategies of cereal cyst nematodes, Heterodera spp., in Turkey. Euphytica 2015, 202, 173-188. [CrossRef]

6. Dababat, A.A.; Fourie, H. Nematode parasites of cereals. Plant Parasitic Nematodes. In Subtropical and Tropical Agriculture; Sikora, R.A., Coyne, D., Hallmann, J., Timper, P., Eds.; CAB International: Wallingford, UK, 2018; pp. 163-221.

7. Rivoal, R.; Cook, R. Nematode pests of cereals. In Plant Parasitic Nematodes in Temperate Agriculture; Evans, K., Trudgill, D.L., Webster, J.M., Eds.; CABI Publishing: Wallingford, UK, 1993; pp. 259-303.

8. Sahin, E.; Nicol, J.M.; Elekcioglu, H.; Rivoal, R. Hatching of Heterodera filipjevi in controlled and natural temperature conditions in Turkey. Nematology 2009, 12, 277-287. [CrossRef]

9. Yan, G.P.; Smiley, R.W. Discovery of Heterodera filipjevi on wheat in the USA. In Cereal Cyst Nematodes: Status, Research and Outlook; Riley, I.T., Nicol, J.M., Dababat, A.A., Eds.; CIMMYT: Ankara, Turkey, 2009; pp. 94-99.

10. Seid, A.; İmren, M.; Ali, M.A.; Toumi, F.; Paulitz, T.; Dababat, A.A. Genetic Resistance of Wheat towards Plant-Parasitic Nematodes: Current Status and Future Prospects. Biotech Stud. 2021, 30, 43-62. [CrossRef]

11. Mokrini, F.; Viaene, N.; Waeyenberge, L.; Dababat, A.A.; Moens, M. Characterization of cereal cyst nematodes (Heterodera spp.) in Morocco based on morphology, morphometrics and rDNA-ITS sequence analysis. J. Plant Prot. Res. 2017, 57, 219-227. [CrossRef]

12. Greco, N.; Di Vito, M. Nematodes of food legumes in the Mediterranean Basin 1. Eppo Bull. 1994, 24, 393-398. [CrossRef]

13. Sabová, M.; Valocká, B.; Lišková, M.; Vargová, V.; Marek, J. Occurrence and distribution of Heterodera avenae Woll, 1924 in the Czech Republic. Ochr. Rostl. 1989, 25, 59-70.

14. Subbotin, S.A.; Rumpenhorst, H.J.; Sturhan, D. Morphological and electrophoretic studies on populations of the Heterodera avenae complex from the former USSR. Russ. J. Nematol. 1996, 4, 29-38.

15. Mokrini, F.; Waeyenberge, L.; Viaene, N.; Moens, M. First report of the cereal cyst nematode Heterodera latipons on wheat in Morocco. Plant Dis. 2012, 96, 774. [CrossRef]

16. Castillo, P.; Vovlas, N. Pratylenchus (Nematoda: Pratylenchidae): Diagnosis, Biology, Pathogenicity and Management; Brill: Leiden, The Netherlands, 2007.

17. Dababat, A.A.; Ferney, G.B.H.; Erginbas-Orakci, G.; Dreisigacker, S.; Imren, M.; Toktay, H.; Ogbonnaya, F. Association analysis of resistance to cereal cyst nematodes (Heterodera avenae) and root lesion nematodes (Pratylenchus neglectus and P. thornei) in CIMMYT advanced spring wheat lines for semi-arid conditions. Breed. Sci. 2016, 66, 692-702. [CrossRef] [PubMed]

18. Forge, T.A.; Larney, F.J.; Kawchuk, L.M.; Pearson, D.C.; Koch, C.; Blackshaw, R.E. Crop rotation effects on Pratylenchus neglectus populations in the root zone of irrigated potatoes in southern Alberta. Can. J. Plant Pathol. 2015, 37, 363-368. [CrossRef]

19. Meskine, M.; Janati, A.; Abbad Andaloussi, F. Résultats préliminaires de l'étude sur les nématodes phytophages associés aux cultures de blé et orge. J. Natl. Phytiatr. 1984, 28-30.

20. Mokrini, F.; Waeyenberge, L.; Viaene, N.; Andaloussi, F.A.; Moens, M. Diversity of root-lesion nematodes (Pratylenchus spp.) associated with wheat (Triticum aestivum and T. durum) in Morocco. Nematology 2016, 18, 781-801. [CrossRef]

21. Doran, J.W. Soil health and global sustainability: Translating science into practice. Agric. Ecosyst. Environ. 2002, 88, 119-127. [CrossRef]

22. Bongers, T. The maturity index: An ecological measure of environmental disturbance based on nematode species composition. Oecologia 1990, 83, 14-19. [CrossRef]

23. Villenave, C.; Ba, A.O.; Rabary, B. Analyse du fonctionnement biologique du sol par l'étude de la nématofaune: Semis direct versus labour sur les hautes terres près d'Antsirabé (Madagascar). Etude Gest. Sols 2009, 16, 369-378.

24. Ferris, H.; Bongers, T.; de Goede, R.G. A framework for soil food web diagnostics: Extension of the nematode faunal analysis concept. Appl. Soil Ecol. 2001, 18, 13-29. [CrossRef]

25. Bongers, T.; van der Meulen, H.; Korthals, G. Inverse relationship between the nematode maturity index and plant parasite index under enriched nutrient conditions. Appl. Soil Ecol. 1997, 6, 195-199. [CrossRef]

26. Yeates, G.W.; Bongers, T. Nematode diversity in agroecosystems. In Invertebrate Biodiversity as Bioindicators of Sustainable Landscapes; Elsevier: Amsterdam, The Netherlands, 1999; Volume 1, pp. 113-135. [CrossRef] 
27. Boag, B.; Yeates, G.W. Soil nematode biodiversity in terrestrial ecosystems. Biodivers. Conserv. 1998, 7, 617-630. [CrossRef]

28. Neher, D.A.; Wu, J.; Barbercheck, M.E.; Anas, O. Ecosystem type affects interpretation of soil nematode community measures. Appl. Soil Ecol. 2005, 30, 47-64. [CrossRef]

29. Wasilewska, L. Soil invertebrates as bioindicators, with special reference to soil-inhabiting nematodes. Russ. J. Nematol. 1997, 5, 113-126.

30. Pankhurst, C.; Doube, B.M.; Gupta, V. Biological Indicators of Soil Health; Oxon-New York; CAB International: Wallingford, UK, 2002.

31. Barker, K.R.; Nusbaum, C.J.; Nelson, L. Effects of storage temperature and extraction procedure on recovery of plant-parasitic nematodes from field soils. J. Nematol. 1969, 1, 240. [PubMed]

32. Hooper, D.J. Extraction of free-living nematode stages from soil. In Laboratory Methods for Work with Plant and Soil Nematodes; Southey, J.F., Ed.; Her Majesty's Stationery Office: London, UK, 1986; pp. 5-22.

33. De Grisse, A.T. Redescription ou modification de quelques techniques utilisés dans l'études dęs nématodes phytoparaires. Meded. Rijksfakulteit Landbouwwet. Gent 1969, 34, 351-356.

34. Mai, W.F.; Lyon, H.H. Pictorial Key to the Genera of Plant-Parasitic Nematodes, 4th ed.; Cornell University Press: Ithaca, NY, USA, 1975.

35. Mai, W.F.; Mullin, P.G. Plant-Parasitic Nematodes: A Pictorial Key to Genera; Cornell University Press: Ithaca, NY, USA, 1996.

36. Brzeski, M.W. Review of the genus Ditylenchus filipjev, 1936 (Nematoda: Anguinidae). Rev. Nématol. 1991, 14, 9-59.

37. Andrássy, I. Free-Living Nematodes of Hungary: Nematoda Errantia; Hungarian Natural History Museum: Budapest, Hungary, 2005; Volume 1.

38. Geraert, E. The Tylenchidae of the World: Identification of the Family Tylenchidae (Nematoda); Academia Press: Ghent, Belgium, 2008.

39. Geraert, E. The Dolichodoridae of the World: Identification of the Family Dolichodoridae (Nematoda); Academia Press: Ghent, Belgium, 2011.

40. Taylor, D.P.; Netscher, C. An improved technique for preparing perineal patterns of Meloidogyne spp. Nematologica 1974, 20, 268-269.

41. Handoo, Z.A. A key and compendium to species of the Heterodera avenae group (Nematoda: Heteroderidae). J. Nematol. 2002, 34, 250.

42. Anderson, J.M.; Ingram, J.S.I. A Handbook of Methods; CAB International: Wallingford, UK, 1993.

43. Richards, L.A. Diagnosis and Improvement Saline and Alkaline Soils; US Department of Agriculture: Washington, DC, USA, 1954.

44. Sims, J.T.; Johnson, G.V. Micronutrient soil tests. Micronutr. Agric. 1991, 4, 427-476. [CrossRef]

45. Allison, L.E. Wet-combustion apparatus and procedure for organic and inorganic carbon in soil. Soil Sci. Soc. Am. J. 1960, 24, 36-40. [CrossRef]

46. Nelson, D.W.; Sommers, L.E. Total carbon, organic carbon, and organic matter. Methods of soil analysis: Part 3. Chem. Methods 1996, 5, 961-1010.

47. Barbano, D.M.; Clark, J.L. Total nitrogen content of milk: A rapid microwave Kjeldahl digestion. J. Dairy Sci. 1990, 73, 83.

48. Boag, B. Standardization of ecological terms in nematology. Fundam. Appl. Nematol. 1993, 16, $190-191$.

49. Krebs, C.J. Ecology: The Experimental Analysis of Distribution and Abundance; Harper and Row: New York, NY, USA, 1985.

50. Ferris, H.; Matute, M.M. Structural and functional succession in the nematode fauna of a soil food web. Appl. Soil Ecol. 2003, 23, 93-110. [CrossRef]

51. Clarke, K.R. Non-parametric multivariate analyses of changes in community structure. Austral Ecol. 1993, 18, 117-143. [CrossRef]

52. Fortuner, R.; Merny, G. Les nématodes parasites des racines associés au riz en Basse-Casamance (Sénégal) et en Gambie. Cah. ORSTOM Série Biol. 1973, 21, 4-43.

53. Sieriebriennikov, B.; Ferris, H.; de Goede, R.G. NINJA: An automated calculation system for nematode-based biological monitoring. Eur. J. Soil Biol. 2014, 61, 90-93. [CrossRef]

54. R Core Team. R: A Language and Environment for Statistical Computing; R Foundation for Statistical Computing: Vienna, Austria, 2020.

55. Yellowbrick: Machine Learning Visualization. Available online: https://www.scikit-yb.org/en/latest/ (accessed on 28 January 2021).

56. Sviewgui 0.3.5. Available online: https://pypi.org/project/sviewgui/ (accessed on 28 January 2021).

57. Bio.Phylo Package. Available online: https://biopython.org/docs/1.75/api/Bio.Phylo.html (accessed on 5 February 2021).

58. NetworkX: Network Analysis in Python. Available online: https://networkx.org (accessed on 5 February 2021).

59. Matplotlib-Venn: Venn Diagram Plotting Routines for Python/Matplotlib. Available online: https://github.com/konstantint/ matplotlib-venn (accessed on 18 February 2021).

60. Matplotlib-Lollipop: Lollipop Chart. Available online: https://www.data-to-viz.com/graph/lollipop (accessed on 18 February 2021).

61. Ordination Methods (skbio.math.stats.ordination). Available online: http://scikit-bio.org/docs/0.1.3/math.stats.ordination.html (accessed on 27 February 2021).

62. Neilsen, M.N.; Winding, A. Microorganisms as Indicators of Soil Health; Technical Report; National Environment Research Institute: Roskilde, Denmark, 2002 
63. Bongers, T.; Ferris, H. Nematode community structure as a bioindicator in environmental monitoring. Trends Ecol. Evol. 1999, 14, 224-228. [CrossRef]

64. Yildiz, S..; Imren, M.; Duman, N. Nematode biodiversity in cereal growing areas of Bolu, Turkey. Turk. Entomol. Derg. 2017, 41, 159-168. [CrossRef]

65. Liu, Y.B.; Li, X.Y.; Liu, Q.Z. Soil nematode communities in jujube (Ziziphus jujuba Mill.) rhizosphere soil under monoculture and jujube/wheat (Triticum aestivum Linn.) intercropping systems, a case study in Xinjiang arid region, northwest of China. Eur. J. Soil Biol. 2016, 74, 52-59. [CrossRef]

66. Diakhaté, S. Influence de L'arbuste Piliostigma Reticulatum (D.C.) Hochst (Caesalpinioideae) sur les Communautés de Microorganismes et de Nématodes d'un sol Cultivé en mil au Sénégal (Nioro). Ph.D. Thesis, Université Cheikh Anta Diop, Dakar, Senegal, 2014.

67. Yeates, G.W. Nematodes as soil indicators: Functional and biodiversity aspects. Biol. Fertil. Soils 2003, 37, 199-210. [CrossRef]

68. Song, M.; Jing, S.; Zhou, Y.; Hui, Y.; Zhu, L.; Wang, F.; Wan, S. Dynamics of soil nematode communities in wheat fields under different nitrogen management in Northern China Plain. Eur. J. Soil Biol. 2015, 71, 13-20. [CrossRef]

69. Didden, W.A.M.; Marinissen, J.C.Y.; Vreeken-Buijs, M.J.; Burgers, S.L.G.E.; De Fluiter, R.; Geurs, M.; Brussaard, L. Soil meso-and macrofauna in two agricultural systems: Factors affecting population dynamics and evaluation of their role in carbon and nitrogen dynamics. Agric. Ecosyst. Environ. 1994, 51, 171-186. [CrossRef]

70. Yeates, G.W.; Bongers, T.; De Goede, R.G.; Freckman, D.W.; Georgieva, S. Feeding habits in soil nematode families and genera-An outline for soil ecologists. J Nematol. 1993, 25, 315. [PubMed]

71. Ferris, H.; Bongers, T. Nematode indicators of organic enrichment. J. Nematol. 2006, 38, 3. [PubMed]

72. Zhao, J.; Neher, D.A. Soil nematode genera that predict specific types of disturbance. Appl. Soil Ecol. 2013, 64, 135-141. [CrossRef]

73. Ogolla, J.A. Diversity of Plant Parasitic Nematodes Associated with Maize (Zea mays L.) and Integrated Control of Lesion Nematodes (Pratylenchus spp.). Ph.D. Thesis, Kenyatta University, Nairobi, Kenya, 2005.

74. Neher, D.A. Ecology of plant and free-living nematodes in natural and agricultural soil. Annu. Rev. Phytopathol. 2010, 48, 371-394. [CrossRef]

75. Freckman, D.W.; Ettema, C.H. Assessing nematode communities in agroecosystems of varying human intervention. Agric. Ecosyst. Environ. 1993, 45, 239-261. [CrossRef]

76. Ruess, L.; Ferris, H. Decomposition pathways and successional changes. Nematol. Monogr. Perspect. 2004, 2, 547-556.

77. Simpson, E.H. Measurement of diversity. Nature 1949, 163, 668. [CrossRef]

78. Nielsen, U.N.; Ayres, E.; Wall, D.H.; Li, G.; Bardgett, R.D.; Wu, T.; Garey, J.R. Global-scale patterns of assemblage structure of soil nematodes in relation to climate and ecosystem properties. Glob. Ecol. Biogeogr. 2014, 23, 968-978. [CrossRef]

79. Godefroid, M.; Tixier, P.; Chabrier, C.; Djigal, D.; Quénéhervé, P. Associations of soil type and previous crop with plant-feeding nematode communities in plantain agrosystems. Appl. Soil Ecol. 2017, 113, 63-70. [CrossRef]

80. Krif, G.; Mokrini, F.; Aissami, A.E.; Laasli, S.E.; Imren, M.; Özer, G.; Dababat, A.A. Diversity and Management Strategies of Plant Parasitic Nematodes in Moroccan Organic Farming and Their Relationship with Soil Physico-Chemical Properties. Agriculture 2020, 10, 447. [CrossRef]

81. Van Diepeningen, A.D.; de Vos, O.J.; Korthals, G.W.; van Bruggen, A.H. Effects of organic versus conventional management on chemical and biological parameters in agricultural soils. Appl. Soil Ecol. 2006, 31, 120-135. [CrossRef]

82. Cadet, P.; Thioulouse, J. Identification of soil factors that relate to plant parasitic nematode communities on tomato and yam in the French West Indies. Appl. Soil Ecol. 1998, 8, 35-49. [CrossRef]

83. Melakeberhan, H.; Maung, Z.; Lee, C.L.; Poindexter, S.; Stewart, J. Soil type-driven variable effects on cover-and rotation-crops, nematodes and soil food web in sugar beet fields reveal a roadmap for developing healthy soils. Eur. J. Soil Biol. 2018, 85, 53-63. [CrossRef]

84. Prot, J.C.; Van Gundy, S.D. Effect of soil texture and the clay component on migration of Meloidogyne incognita second-stage juveniles. J. Nematol. 1981, 13, 213.

85. Thompson, J.P.; Clewett, T.G.; Sheedy, J.G.; Reen, R.A.; O'Reilly, M.M.; Bell, K.L. Occurrence of root-lesion nematodes (Pratylenchus thornei and P. neglectus) and stunt nematode (Merlinius brevidens) in the northern grain region of Australia. Australas. Plant Pathol. 2010, 39, 254-264. [CrossRef]

86. Mokrini, F.; Laasli, S.E.; Karra, Y.; El Aissami, A.; Dababat, A.A. Diversity and incidence of plant-parasitic nematodes associated with saffron (Crocus sativus L.) in Morocco and their relationship with soil physicochemical properties. Nematology 2019, 22, 87-102. [CrossRef]

87. Georgieva, S.S.; McGrath, S.P.; Hooper, D.J.; Chambers, B.S. Nematode communities under stress: The long-term effects of heavy metals in soil treated with sewage sludge. Appl. Soil Ecol. 2002, 20, 27-42. [CrossRef]

88. Oteifa, B.A. Nitrogen source of the host nutrition in relation to infection by a root-knot nematode, Meloidogyne incognita. Plant Dis. Report. 1955, 39, 902-903.

89. Widmer, T.L.; Mitkowski, N.A.; Abawi, G.S. Soil organic matter and management of plant-parasitic nematodes. J. Nematol. 2002, 34, 289. [PubMed]

90. Hominick, W.M.; Hunt, D.J.; Reid, A.; Briscoe, B.R.; Bohan, D.A. Biosystematics, phylogeny, and population genetics of entomopathogenic nematodes. In Taxonomy, Phylogeny and Gnotobiological Studies of Entomopathogenic Nematode Bacterium Complexes; Boemare, N., Richardson, P., Coudert, F., Eds.; European Commission Publication: Brussels, Belgium, 1999 ; pp. 45-53. 
91. Hu, C.; Qi, Y. Effective microorganisms and compost favor nematodes in wheat crops. Agron. Sustain. Dev. 2013, 33, 573-579. [CrossRef]

92. Barros, P.Â.; Pedrosa, E.M.R.; de Oliveira Cardoso, M.S.; Rolim, M.M. Relationship between soil organic matter and nematodes in sugarcane fields. Semin. Cienc. Agrar. 2017, 38, 551-559. [CrossRef]

93. Papatheodorou, E.M.; Argyropoulou, M.D.; Stamou, G.P. The effects of large-and small-scale differences in soil temperature and moisture on bacterial functional diversity and the community of bacterivorous nematodes. Appl. Soil Ecol. 2004, 25, 37-49. [CrossRef]

94. Berry, S.; Rhodes, R. Green manure crops: Agronomic characteristics and effect on nematodes. In Proceedings of the 80th Annual Congress of the South African Sugar Technologists' Association, Durban, South Africa, 18-20 July 2006; South African Sugar Technologists' Association: Durban, South Africa, 2006; pp. 269-273.

95. Ferris, H. Form and function: Metabolic footprints of nematodes in the soil food web. Eur. J. Soil Biol. 2010, 46, 97-104. [CrossRef]

96. Imren, M.; Ciftci, V.; Yildiz, S..; Kutuk, H.; Dababat, A.A. Occurrence and population dynamics of the root lesion nematode Pratylenchus thornei (Sher and Allen) on wheat in Bolu, Turkey. Turk. J. Agric. For. 2017, 41, 35-41. [CrossRef]

97. Bird, A.F. Plant Response to Root-Knot Nematode. Annu. Rev. Phytopathol. 1974, 12, 69-85. [CrossRef]

98. Boag, B. Effects of temperature on rate of feeding of the plant parasitic nematodes Rotylenchus robustus, Xiphinema diversicaudatum, and Hemicycliophora conida. J. Nematol. 1980, 12, 193.

99. Duyck, P.F.; Dortel, E.; Tixier, P.; Vinatier, F.; Loubana, P.M.; Chabrier, C.; Quénéhervé, P. Niche partitioning based on soil type and climate at the landscape scale in a community of plant-feeding nematodes. Soil Biol. Biochem. 2012, 44, 49-55. [CrossRef]

100. Pritchard, S.G. Soil organisms and global climate change. Plant Pathol. 2011, 60, 82-99. [CrossRef]

101. Riley, I.T.; Kelly, S.J. Endoparasitic nematodes in cropping soils of Western Australia. Aust. J. Exp. Agric. 2002, 42, 49-56. [CrossRef]

102. Kandel, S.L.; Smiley, R.W.; Garland-Campbell, K.; Elling, A.A.; Huggins, D.; Paulitz, T.C. Spatial distribution of root lesion nematodes (Pratylenchus spp.) in a long-term no-till cropping system and their relationship with soil and landscape properties. Eur. J. Soil Biol. 2018, 150, 1011-1021. [CrossRef] 